\title{
Automated Layerwise Detection of Geometrical Distortions in Laser Powder Bed Fusion
}

\author{
Luca Pagani ${ }^{\mathrm{a}}$, Marco Grasso ${ }^{\mathrm{b}, *}$, Paul J Scott ${ }^{\mathrm{a}}$, Bianca M Colosimo ${ }^{\mathrm{b}}$ \\ ${ }^{a}$ EPSRC Future Metrology Hub, University of Huddersfield, Huddersfield, Queensgate, HD13DH, UK \\ ${ }^{b}$ Department of Mechanical Engineering, Politecnico di Milano, via La Masa 1, 20156, Milan, Italy
}

\begin{abstract}
In-situ layerwise imaging in laser powder bed fusion (L-PBF) has been implemented by many system developers to monitor the powder bed homogeneity. Increasing attention has been recently devoted to the possibility of using the same sensing approach to detect also in-plane and out-of-plane geometrical distortions of the part while it is being produced. To this aim, seminal works investigated the suitability of various image segmentation algorithms and assessed the accuracy of layerwise dimensional and geometrical measurements. Nevertheless, there is a lack of automated methods to identify, in-situ and in-process, geometrical defects and out-of-control deviations from the nominal geometry. This study presents a methodology that combines an active contours methodology for image segmentation with a statistical process monitoring approach suitable to deal with complex geometries that change layer by layer. The proposed approach enables a data-driven and automated alarm rule to detect the onset of geometrical distortions during the build by comparing the slice contour reconstruction with the nominal geometry in each layer. Moreover, by coupling edge-based and region-based segmentation techniques, the method is sufficiently robust to be applied to imaging and illumination setups that are already available on industrial L-PBF systems. The effectiveness of the proposed approach was tested on a real case study involving the L-PBF of complex Ti6Al4V parts that exhibited local geometrical distortions.
\end{abstract}

Keywords: Additive manufacturing, image segmentation, in-situ monitoring, geometrical distortion

\section{Introduction}

The layerwise production paradigm in powder bed fusion processes enables the opportunity to gather a large amount of data while the part is being produced, which can be used to support qualification procedures and to anticipate the detection of defects and unstable process states [1,2]. In laser powder bed fusion (L-PBF), it is possible to measure several quantities of interest at different levels and with different sensing methods [3]. The first level regards the acquisition of images of the entire powder bed, possibly before and after each recoating. A second level regards the measurement of so-called "process signatures" during the melting phase of each scanned track.

${ }^{*}$ Corresponding author, marcoluigi.grasso@polimi.it 
This usually entails high-speed video imaging methods to capture fast and transient phenomena related to the cooling history or the ejection of process by-products. One last level regards the monitoring of the salient melt pool properties, which is commonly achieved by using co-axial sensing methods exploiting the optical path of the laser. This study focuses on the first level.

In this framework, increasing attention has been devoted to the use of layerwise images of the powder bed either to identify recoating errors and powder bed in-homogeneities $[4,5,6]$ or to identify surface and geometrical defects in the printed area $[7,8,9,10,11,12,13]$. Some of these methods are particularly appealing for a direct industrial implementation, as in most cases they exploit in-situ sensing architectures that are either already available in industrial LPBF systems or easy to integrate [3]. Various authors pointed out that powder bed images acquired after the laser scan of each layer could be used to detect both in-plane and out-of-plane defects. Various seminal studies focused on the identification of recoating errors and powder bed inhomogeneities by using layerwise images gathered after the powder recoating operation $[4,5,6]$, and some of these methods are already implemented by most L-PBF system developers. Another stream of research regards the detection of out-of-plane irregularities in the printed area, like so-called super-elevated edges, and the measurement of the surface topography of the slice $[9,10,14,15,16,17,18,19]$. This stream is motivated by the fact that irregular surface patterns may introduce an undesired variability in the local powder thickness, affecting the actual energy density provided to the material. Surface irregularities may also interfere with the recoating operation, producing additional defect propagations within the build area.

This study focuses on a different problem, namely the in-situ detection and characterization of in-plane geometrical deviations. In this framework, the seminal study of Foster et al. [4] demonstrated the feasibility of in-situ 3D reconstruction of the part geometry by segmenting layerwise images. As pointed out by Caltanissetta et al. [12], part dimensions and geometries measured insitu may be not representative of the final dimensions and geometry of the as-built part, as some deviations, including shrinkage and thermal stress-induced distortions, may be not captured on a layer-by-layer basis. However, when a major departure from the nominal shape is observed in one layer, it is worth signalling as soon as possible, since it may indicate a defect that cannot be recovered as the process goes on.

Aminzadeh [20], Aminzadeh and Kurfess [11] investigated the accuracy of in-situ contour detection in L-PBF by comparing the identified contours against a manual segmentation applied to the same images. The segmentation approach proposed by the authors combined histogram-based thresholding with image pre-filtering and morphological operations. Caltanissetta et al. [12] presented an in-situ measurement performance characterization analysis based on a different family of image segmentation methods, i.e., active contours [21]. Caltanissetta et al. [12] used ex-situ optical measurements as ground truth to determine the accuracy of layerwise contour detection. They pointed out that the pure measurement error was up to one order of magnitude lower than the total measurement variability affected by part-to-part and build-to-build variations, showing that in-situ reconstructions could be adequate for macro-geometrical distortion detection.

Other authors combined contour detection with surface pattern analysis for out-of-plane defect detection and for layerwise topography reconstruction. Abdelrahman et al. [22] proposed a method to automatically detect surface anomalies in layerwise images related to uneven surface patterns within the laser printed area. They applied the active contours algorithm to register the nominal slice contour to in-situ images in a pre-processing phase. Li et al. [8] proposed the active contours methodology to identify the region of interest consisting of the printed area within the layer, and then a topography map was estimated via fringe projection coupled with stereo imaging within that region. 
In all the aforementioned studies, an accurate contour detection of the printed slice was needed, either to directly determine the presence of in-plane distortions or to identify the region of interest for the following application of surface pattern analysis methods. In this framework, one open issue consists of the lack of effective and robust methods for contour detection of complex geometries in L-PBF layerwise images. Indeed, the seminal studies that investigated the accuracy of in-situ reconstructed contours involved simple and layerwise invariant shapes [12, 11]. As shown in Figure 1, segmentation methods previously proposed in the literature [12, 22] may fail in the presence of more complicated geometries and images gathered with industrial settings. Figure 1A shows a layerwise image acquired with the powder bed camera installed on an EOS M290 during the production of a complex shape. Figure 1B and Figure 1C show, respectively, the slice contour reconstruction with the region-based methods proposed by Abdelrahman et al. [22] and Caltanissetta et al. [12]. In both cases, the contour reconstruction is poorly representative of the real slice geometry, especially in critical features like thin walls. As a matter of fact, layerwise images in L-PBF exhibit several challenges, e.g., noisy patterns, non-homogeneous pixel intensity patterns and not well-defined edges of foreground areas, which may limit the performances of image segmentation techniques, including traditional active contours-based methods.
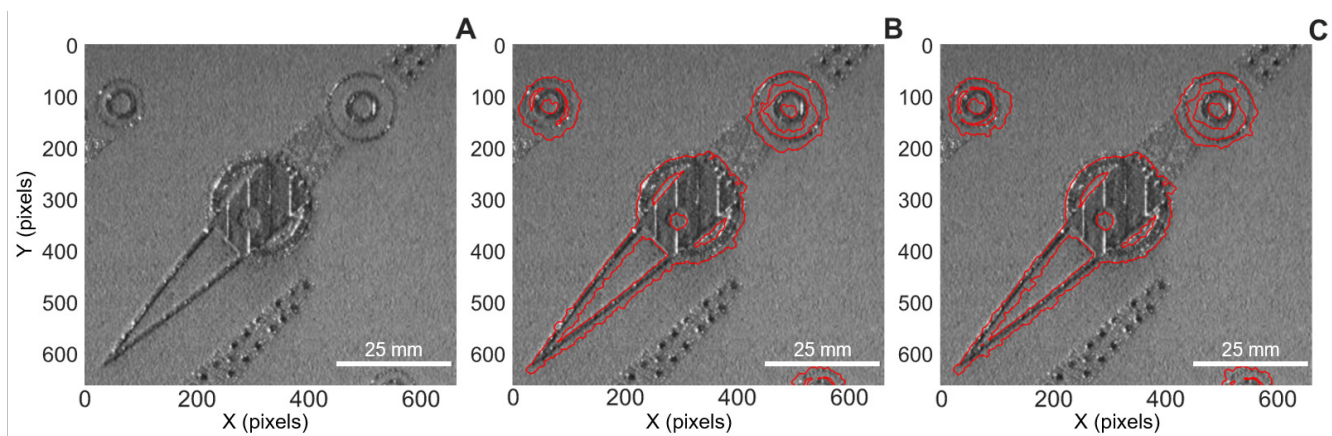

Figure 1: Original layerwise image acquired on a EOS M290 (A), contour reconstruction by means of the active contours algorithm used in Abdelrahman et al. [22] (B), contour reconstruction by means of the active contours algorithm used in Caltanissetta et al. [12] (C)

A further open issue regards the lack of methods to automatically identify and signal a deviation between the in-situ reconstructed contours and the nominal geometry of the slice. Although control charts have been proposed in previous studies to monitor the powder bed homogeneity [6], there is a lack of such tools for in-line monitoring of the printed geometry. The contribution of the present study is aimed at specifically filling this gap by proposing a novel methodology that combines a robust image segmentation approach with a statistical process monitoring technique for automated in-situ detection of geometrical distortions. The proposed approach relies on an active contours formulation that combines the benefits of both edge-based and region-based segmentation methods [23] instead of relying only on region-based descriptors as done in previous studies mentioned above. The inclusion of local pixel intensity gradients into the active contours energy minimization function is expected to make the image segmentation more robust to illumination conditions and machine vision equipment available in industrial systems and more suitable to deal with complicated geometries. In-situ reconstructed contours are then aligned and compared against the nominal geometry of the slice leading to a deviation map that can be synthesized into a univariate deviation metric. A control charting scheme is then applied to this 
metric to automatically signal any out-of-control geometrical distortion by taking into account the natural layer-by-layer variability of the deviation measurements. To this aim, an adaptive control chart is proposed to deal with geometries that continuously change from one layer to another.

The method was tested on a EOS M290 by using the powder bed imaging already available in the system. An experimental study was carried out by producing a complicated geometry with different orientation and support configurations, to induce the occurrence of geometrical errors and demonstrate their in-situ detectability. A further experimentation, involving simpler specimens, was performed to characterize the performances of the proposed approach in terms of false alarm rate.

Section 2 presents a motivating example that is used as real case study to illustrate and test the method. Section 3 presents the proposed methodology. Section 4 describes the achieved results and Section 5 concludes the paper.

\section{Test case}

An experimental study was carried out by producing a specimen specifically designed to include various complex geometrical features like thin walls, acute corners, massive parts with internal holes, overhang regions, etc. This kind of specimen was selected as it allowed us not only to test the suitability of the proposed approach in the presence of a complex geometry that evolves layer by layer, but also to induce distortions in the part as a consequence of the geometry itself and its orientation within the build. Indeed, the case study was conceived to assess the performances of the proposed approach in the presence of both in-control and out-of-control (defective) shapes. Figure 2 (left panel) shows the test specimen in two different orientations with the corresponding supports. Orientation 2 required a much larger support area than orientation 1, and it was also expected to be more critical in terms of possible geometrical distortions. Figure 2 (right panel) shows how different replicates of the test specimen were placed in the $250 \times 250 \mathrm{~mm}$ build area of an EOS M290 L-PBF system. Orientations 1 and 2 were replicated three times (different replicates were indicated with the capital letters A, B and C). Three additional replicates of the test specimen, namely $3 \mathrm{~A}, 3 \mathrm{~B}$ and $3 \mathrm{C}$, were included into the build with a $45^{\circ}$ orientation. In this study, only parts with orientation 1 and 2 were included into the analysis, as they were the only ones that exhibited geometrical distortions at the end of the process. However, additional results are made available by the authors upon request.

The build was produced by using a Ti6A14V ELI gas atomized powder with average grain size lower than $45 \mu \mathrm{m}$ supplied by TLS Technik. An EOS M290 equipped with an embedded powder bed camera mounting a 1280 x 1024 pixels sensor was used. Default process parameters defined by the AM service bureau that hosted the experimentation for the production of Ti6Al4V parts with this L-PBF system were applied.

Figure 3 shows a scheme of the placement of the powder bed camera and the low angle side illumination source already available in the machine. The same embedded camera of the EOS M290 was used in Scime and Beuth [9] for anomaly detection in the pixel intensity patterns of layerwise images. With this configuration, after perspective correction, cropping and rescaling, layerwise images of pixel size $125 \mu \mathrm{m} /$ pixel were obtained. It is worth noticing that a spatial resolution in the order $100-400 \mu \mathrm{m} / \mathrm{pixel}$ is representative of layerwise imaging implementations in industrial L-PBF systems, but lower than the one achieved in other studies that exploited adhoc prototype systems or external sensor integration into an existing machine [3].

The available illumination configuration on the EOS M290 is appropriate for the detection of 

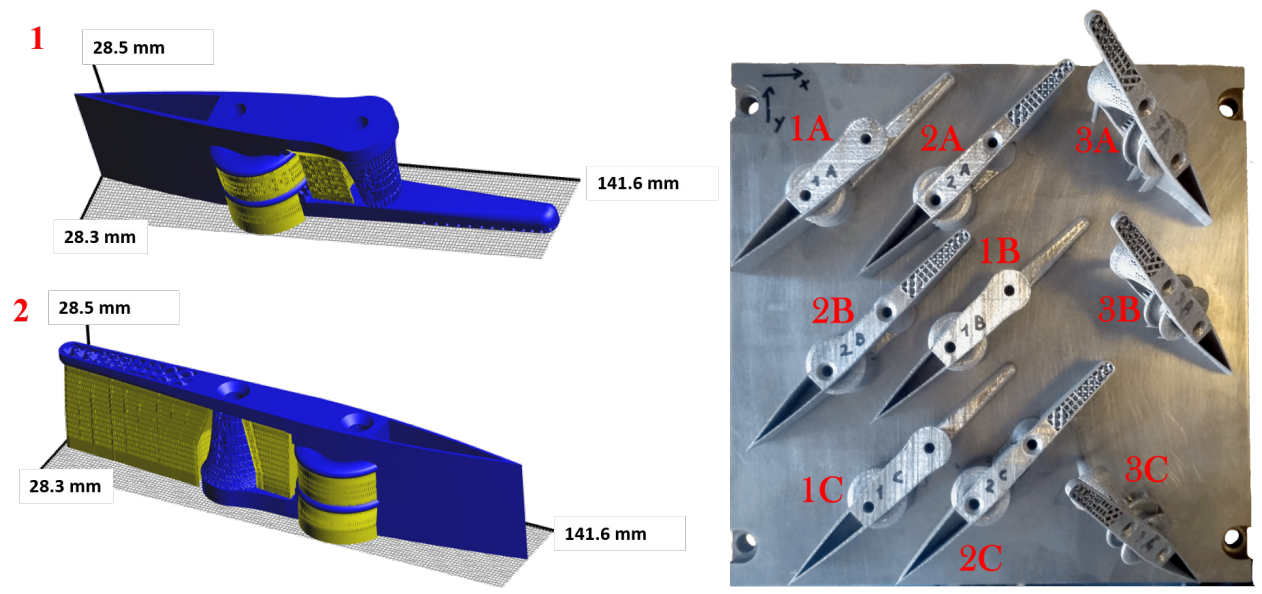

Figure 2: Designed parts and their placement within the build area

out-of-plane distortions and irregularities in the powder bed thanks to the low angle from the baseplate, but it is not necessarily the best illumination condition for image segmentation [12]. These experimental settings were chosen in order to determine the suitability and robustness of the proposed approach in a real industrial environment. Improved image segmentation and insitu detection of geometrical distortions are expected if ad-hoc sensing and illumination sources are used. However, in that case, a modification of the L-PBF system hardware is needed.
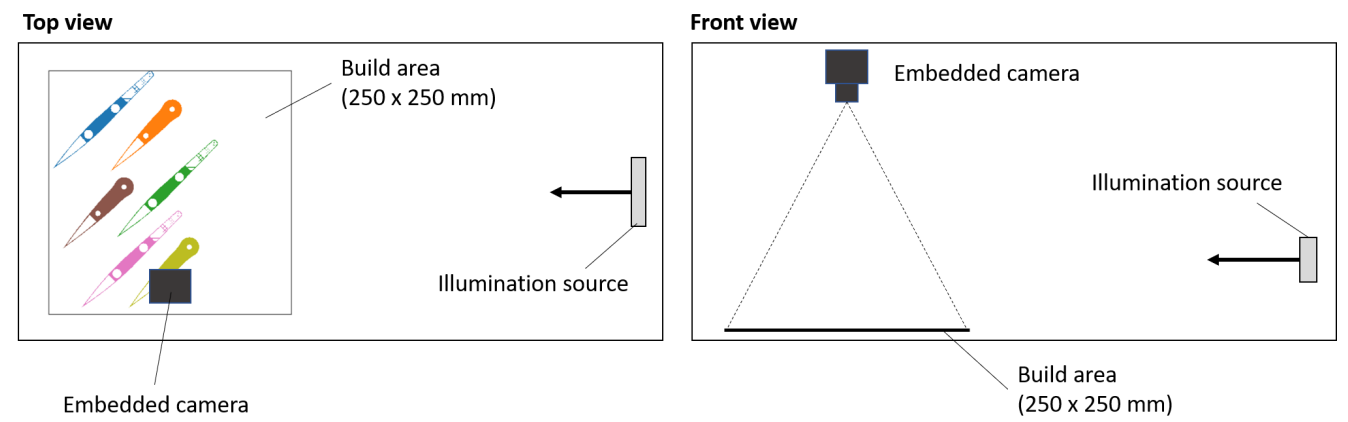

Figure 3: Powder bed camera placement

\section{Methodology}

Figure 4 shows the scheme of the proposed methodology, which includes four major steps, i.e., image pre-processing, image segmentation, deviation map estimation and statistical monitoring of the deviation from the nominal geometry. The following sub-sections describe these four steps with examples from the case study introduced in Section 2. 


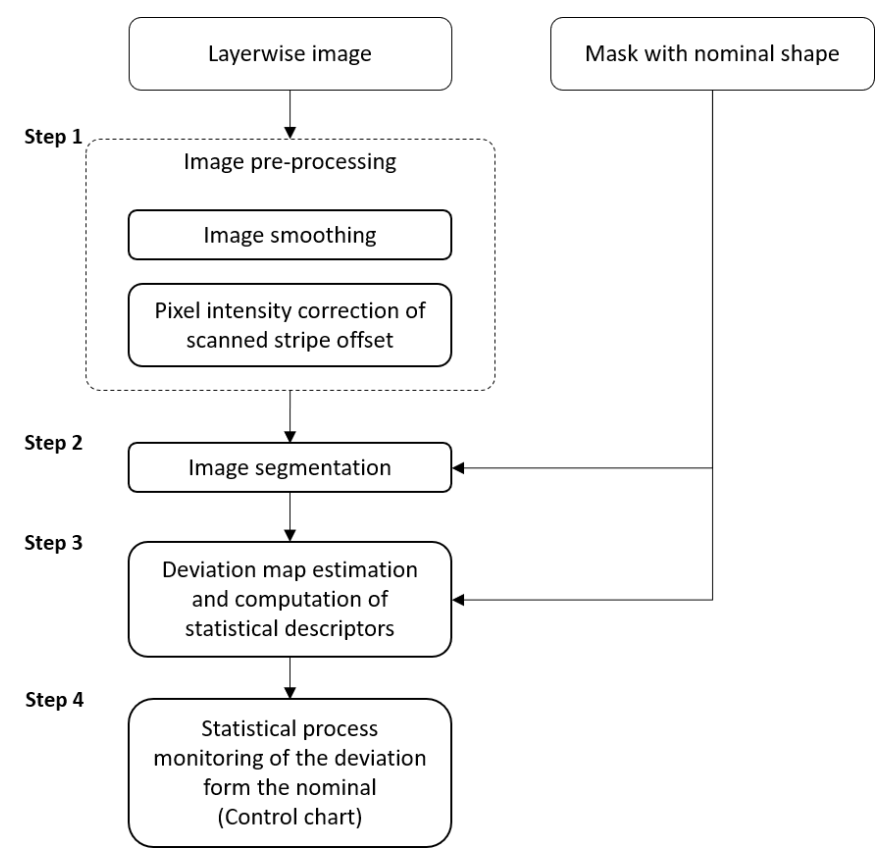

Figure 4: Flowchart of the proposed methodology

\subsection{Image pre-processing}

The layerwise image acquired at the end of the L-PBF of the current layer and before the recoating of the next layer needs to be pre-processed in order to enhance the results in following segmentation and analysis steps. In this study, it is assumed that the camera perspective correction and the alignment between in-situ images and the nominal mask of the slice in each layer are performed during the camera calibration phase. When embedded cameras available in LPBF systems for powder bed imaging are used, the output images made available to the user are usually already calibrated and aligned. If not, standard camera calibration procedures and image alignment algorithms may be applied. The reader is referred to Szeliski [24], Avants et al. [25] for an introduction to these methods. Figure 5 shows an example of layerwise image (after camera perspective correction), the corresponding mask showing the nominal shape of the printed slice and their superimposition, which highlights the proper alignment between the image and the mask.

In order to enhance the identification of the contours between the foreground (the slices scanned by the laser) and the background (the powder bed) a smoothing operation is usually needed, which improves the estimation of local intensity gradients and filters out possible outliers. In this study, the layerwise images were smoothed by means of a Gaussian filter with $\sigma$ equal to 1 , in both directions. A comparison between the original image (a detail) and the smoothed image is shown in Figure 6B.

As far as layerwise L-PBF images are concerned, one additional pre-processing step may be needed depending on the scanning strategy adopted for the production of the part. If the so-called "stripe scan" or "island scan" methods are applied, an offset is left between adjacent stripes or islands (see the highlighted regions in Figure 6). This typically results in a discontinuity of 

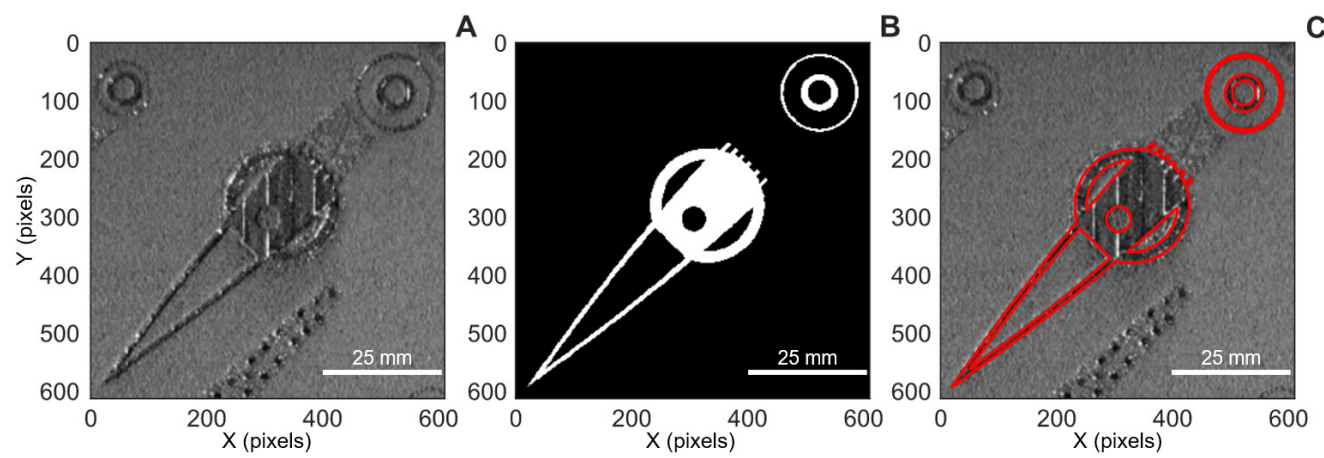

Figure 5: Example of an acquired image (A) and its nominal mask (B), and the contour of the mask superimposed on the original image $(\mathrm{C})$
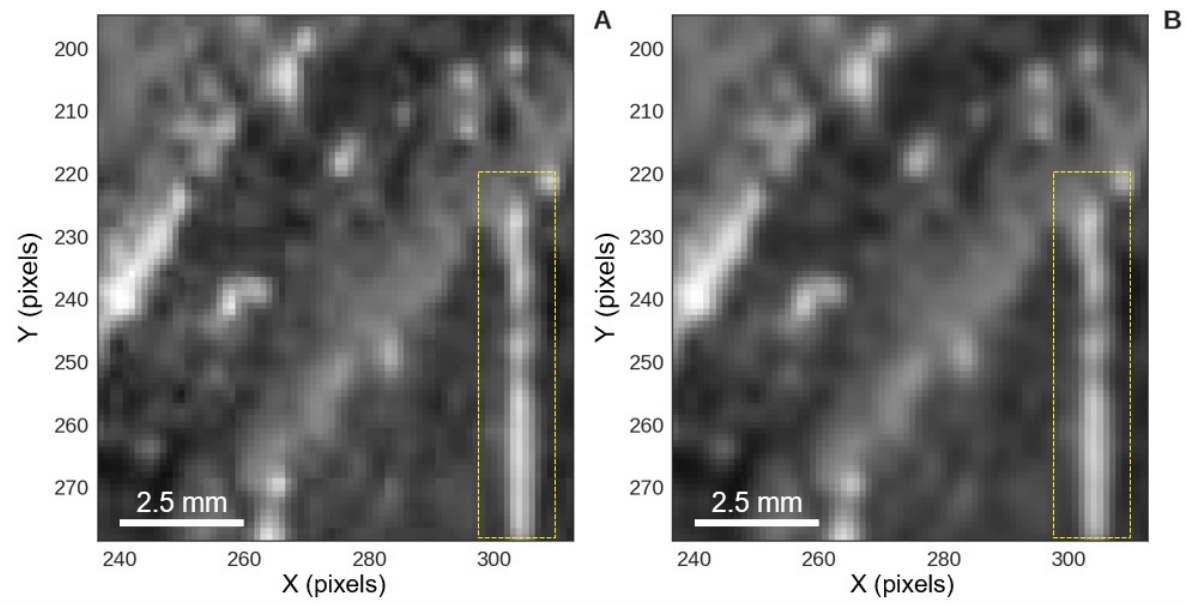

Figure 6: Detail of one original image (A) and corresponding smoothed image (B); the yellow box identifies a discontinuity between two adjacent stripes

pixel intensities within the foreground area along the borders of the stripes or the islands. These internal discontinuities are visible in Figure 6, since the stripe scan strategy was used to produce the specimens. If traditional edge detection and image segmentation methods are applied to an image like the one shown in Figure 5, the offset lines could be identified as contours of the segmented region, leading to a local mis-detection of the actual contours of interest. To avoid this effect, the following approach, which exploits a local pixel intensity correction to "mask" the presence of stripe discontinuities in the image, is proposed. The underlying idea consists of identifying the regions of interest corresponding to these border lines and replace the original intensity of the pixels with a constant intensity value closer to the average intensity of the foreground area. This mitigates the presence of abrupt gradient changes within the scanned area and improves the following image segmentation result. In this phase, the nominal mask is used to define a preliminary identification of the foreground area where the stripe offset correction has to be applied. The first step consists of finding an estimation of the probability density function 
(PDF) of the pixel intensity values inside the nominal mask. The kernel density estimation [26] was used to this aim. An example of the estimated density function, $p(t)$, is shown in Figure 7. A Gaussian kernel with bandwidth set to $1 \%$ of the range of the available values was used. A sensitivity analysis with respect to this parameter is reported in Appendix A. In order to split
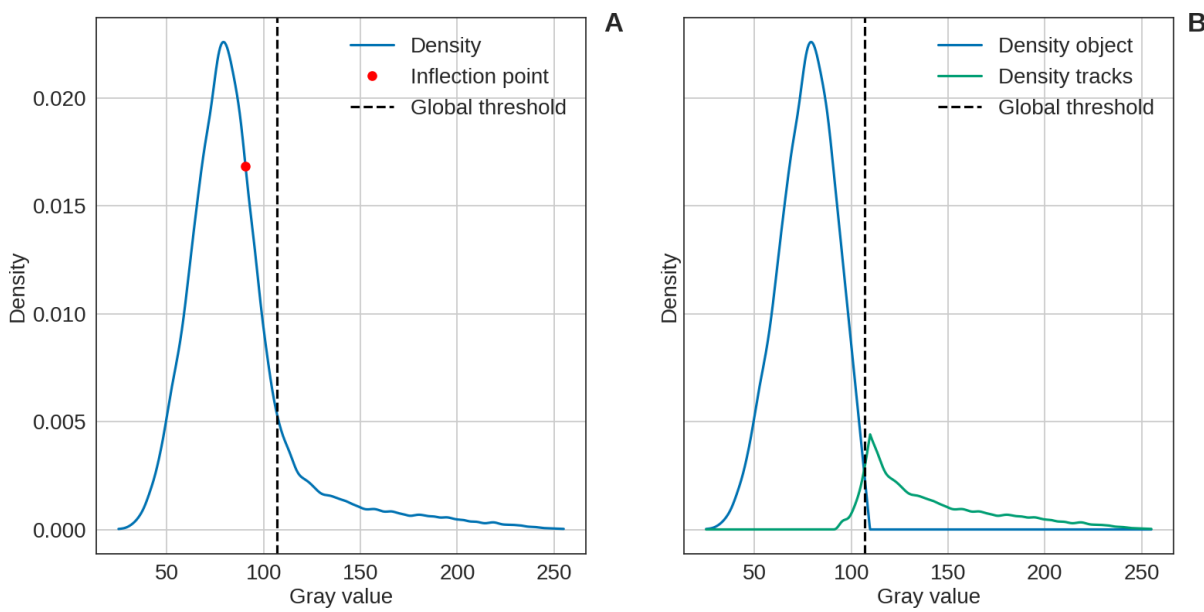

Figure 7: Example of an estimated kernel density function of the pixels within the nominal mask (A), and computed density functions of the dark and bright pixels within the same region, with the indication of the global threshold (B)

the PDF into dark and bright levels, the minimum of the first derivative of the estimated PDF, denoted as "infl" (since it is an inflection point), is computed as:

$$
t_{\text {infl }}=\underset{t}{\arg \min } \frac{d p(t)}{d t}=\underset{t}{\arg \min } p^{\prime}(t)
$$

This inflection value in the example shown in Figure 7 is indicated by a red dot. Then, the estimation of the density curve of dark pixel intensities is computed as a linear approximation of the PDF on the left side of the inflection point:

$$
p_{\text {dark }}(t)=\left\{\begin{array}{ll}
p(t) & \text { if } t \leq t_{\text {infl }} \\
\max \left\{0, p^{\prime}\left(t_{\text {inf }} \cdot\left(t-t_{\text {infl }}\right)+p\left(t_{\text {infl }}\right)\right\}\right. & \text { if } t>t_{\text {infl }}
\end{array} .\right.
$$

The density of bright pixel intensities can be computed as $p_{\text {bright }}(t)=p(t)-p_{\text {dark }}(t)$, instead. The estimated densities are shown in Figure 7B. It should be noted that, since the integrals of the density curves of dark and bright pixel intensities are not equal to one, these curves are not probability functions.

A global threshold, $t_{t h}$, corresponding to the dashed dark line in Figure $7 \mathrm{~B}$, is finally used to segment the region within the nominal mask into two areas, one corresponding to dark pixels and one corresponding to bright pixels. This threshold can be set at the zero of the function $p_{\text {dark }}(t)-p_{\text {bright }}(t)$.

Figure 8A highlights the areas of the image corresponding to bright pixels according to the segmentation based on the aforementioned global threshold. The original intensity of all the pixels belonging to the bright areas is then replaced by a gray value equal to the quantile of order 0.1 computed using the pixels intensity of the original image within the nominal mask. This results 

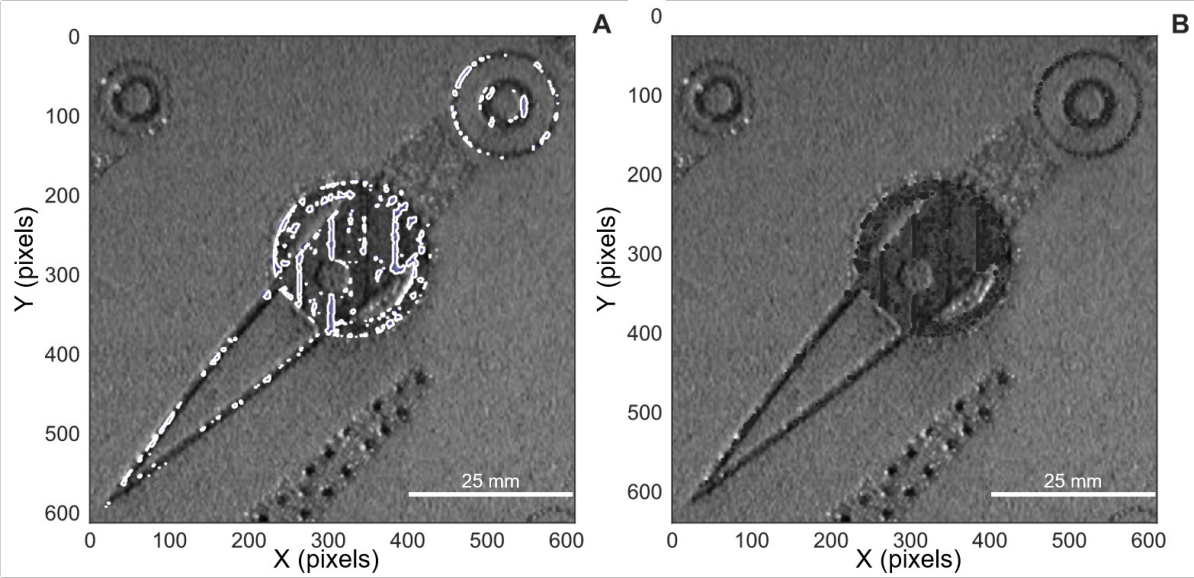

Figure 8: Isolation of bright areas in the foreground region (A) and resulting image after the proposed pixel intensity correction operation (B)

into the image shown in Figure 8B. It is worth mentioning that the isolation of bright pixels might include not only the stripe borders, but also sparse bright pixels within the scanned area and along its contours. Changing the intensity of these sparse pixels by means of the pixel intensity correction operation here proposed is expected to further improve the segmentation results, as it avoids abrupt intensity changes in the region of interest.

The choice of the quantile to determine the replacement value for selected pixels can be customized and tuned depending on the illumination conditions and the pixel intensity patterns in the available images.

\subsection{Image segmentation}

Once the image pre-processing has been completed, the segmentation of the foreground area corresponding to the printed slice is applied. Previous studies $[12,22,8]$ showed that, among all the possible methods that can be applied to this aim, the ones based on active contours are suitable to deal with the specific challenges imposed by layerwise images in L-PBF. Active contours are iterative segmentation methods: starting from a first boundary definition in the form of a closed curve, the shape of this boundary is iteratively changed and adapted by applying shrink/expansion operations called "contour evolution" driven by the minimization of an energy functional. In LPBF images, the nominal geometry of the slice (i.e., the nominal mask) can be used as starting boundary. The level set formulation [27] is then used to solve the active contour algorithm. In the literature, active contours methods for image segmentation can be divided into two main classes: edge- and region-based methods [28]. Edge-based methods work by finding discontinuities in the intensity of an image, where an edge consists of a boundary between two regions characterized by different properties (e.g. pixel intensities). Region-based techniques, instead, exploit a certain region descriptor to guide the contour evolution. Relying on local derivative estimation, edgebased methods may be less effective than region-based ones in the presence of weak region boundaries and noisy patterns that are common in L-PBF layerwise images. On the contrary, edge-based methods are more accurate than region-based ones if the starting contour is close to the true one, as typically happens in L-PBF layerwise images not affected by defects. 
All previous studies in L-PBF applied different variants of region-based level set methods [12, $22,8]$. A different approach was proposed by Liu and Peng [23], Soomro et al. [28], i.e. an active contours approach that allows one to balance both intensity gradients and region descriptors in the estimation of the contours. Since it combines the benefits of both edge-based and regionbased methods, it is believed to be more effective in segmenting noisy and low contrast images like the ones acquired in L-PBF. Moreover, combining the potentials of the two methods it is also believed to be more appropriate to deal with complicated nominal geometries that change from one layer to another. The working principle of the method is briefly reviewed hereafter. Additional details are provided in Soomro et al. [28]. For a review of region-based methods applied in previous studies the reader is referred to Caltanissetta et al. [12].

The contour is represented as the zero level set of a signed distance function, $\varphi(\boldsymbol{x})$, which is defined as:

$$
\varphi(\boldsymbol{x})= \begin{cases}-d(\boldsymbol{x}, \partial \Omega) & \text { if } \boldsymbol{x} \in \Omega \\ d(\boldsymbol{x}, \partial \Omega) & \text { if } \boldsymbol{x} \in \bar{\Omega}\end{cases}
$$

where $d(\boldsymbol{x}, \partial \Omega)$ is the distance between any pixel $\boldsymbol{x}$ in the image and the boundary $\partial \Omega$ of the foreground region $\Omega$, whereas $\bar{\Omega}$ is the negation of $\Omega$, i.e. the domain of pixels outside the foreground region. Let $\varphi(x, t=0)$ be an initial signed distance at iteration $t=0$ computed using the nominal shape of the slice. Starting from the nominal slice contour, the contour evolves according to:

$$
\begin{aligned}
\frac{\partial \varphi(\boldsymbol{x}, t)}{\partial t}= & \delta_{\varepsilon}(\varphi(\boldsymbol{x}))\|\boldsymbol{\nabla} \varphi(\boldsymbol{x})\|\{-w g(\|\boldsymbol{\nabla} I(\boldsymbol{x})\|) \\
& \cdot\left[\iint_{\Omega} K(\|\boldsymbol{x}-\boldsymbol{y}\|)\left|I(\boldsymbol{x})-f_{1}(\boldsymbol{y})\right|^{2} d \boldsymbol{y}\right. \\
& \left.-\iint_{\Omega} K(\|\boldsymbol{x}-\boldsymbol{y}\|)\left|I(\boldsymbol{x})-f_{2}(\boldsymbol{y})\right|^{2} d \boldsymbol{y}\right] \\
& +(1-w)\left[g(\|\nabla I(\boldsymbol{x})\|) \operatorname{div}\left(\frac{\boldsymbol{\nabla} I(\boldsymbol{x})}{\|\boldsymbol{\nabla} I(\boldsymbol{x})\|}\right)\right. \\
& \left.\left.+\boldsymbol{\nabla} g(\|\boldsymbol{\nabla} I(\boldsymbol{x})\|) \cdot \frac{\boldsymbol{\nabla} I(\boldsymbol{x})}{\|\boldsymbol{\nabla} I(\boldsymbol{x})\|}\right]\right\}
\end{aligned}
$$

where $I(\boldsymbol{x})$ is the intensity of the pixel, $f_{1}(\boldsymbol{x})$ and $f_{2}(\boldsymbol{x})$ are local terms representing respectively the inside and the outside of the segmented region at iteration $t$ :

$$
\begin{aligned}
f_{1}(\boldsymbol{x}) & =\frac{K(\boldsymbol{y}) *\left[H_{\varepsilon}(\varphi(\boldsymbol{x})) g(\|\nabla I(\boldsymbol{x})\|) I(\boldsymbol{x})\right]}{K(\boldsymbol{y}) *\left[H_{\varepsilon}(\varphi(\boldsymbol{x})) g(\|\boldsymbol{\nabla} I(\boldsymbol{x})\|)\right]} \\
f_{2}(\boldsymbol{x}) & =\frac{K(\boldsymbol{y}) *\left[\left(1-H_{\varepsilon}(\varphi(\boldsymbol{x}))\right) g(\|\nabla I(\boldsymbol{x})\|) I(\boldsymbol{x})\right]}{K(\boldsymbol{y}) *\left[\left(1-H_{\varepsilon}(\varphi(\boldsymbol{x}))\right) g(\|\boldsymbol{\nabla} I(\boldsymbol{x})\|)\right]}
\end{aligned}
$$

where $K(t)$ is a kernel function: in this study, a dense stencil of width $2 r+1$ was used. $\delta_{\varepsilon}(x)$ is the Dirac delta function and $H_{\varepsilon}(t)$ is the Heaviside step function:

$$
\begin{aligned}
\delta_{\varepsilon}(x) & =\frac{1}{\pi} \frac{\varepsilon}{\varepsilon^{2}+x^{2}} \\
H_{\varepsilon}(x) & =\frac{1}{2}\left[1+\frac{2}{\pi} \arctan \left(\frac{x}{\varepsilon}\right)\right]
\end{aligned}
$$


$\boldsymbol{\nabla} I(\boldsymbol{x})$ and $\|\nabla I(x)\|$ are the gradients of the image pixel intensities and their magnitude respectively, whereas $g(x)$ is a function that converts the norm of the gradient into a so-called "speed" function:

$$
\begin{aligned}
\nabla I & =\left(\frac{\partial I}{\partial x}, \frac{\partial I}{\partial y}\right)^{T} \\
\|\nabla I\| & =\sqrt{\left(\frac{\partial I}{\partial x}\right)^{2}+\left(\frac{\partial I}{\partial y}\right)^{2}} \\
g(x) & =\left(x_{\max }-x_{\min }\right) \frac{1}{1+e^{-\frac{x-\beta}{\alpha}}}+x_{\min } .
\end{aligned}
$$

The speed function $g(x)$ assumes small values in the edges and higher values elsewhere. This allows a correct "flow" of the contour evolution along the successive iterations, where the edges are used as attractors. In this study, the sigmoid function was used in the computation of the speed function, given the gradient magnitude. $x_{\min }$ and $x_{\max }$ are the minimum and maximum, respectively, of the gradient magnitude, while $\alpha$ and $\beta$ are two coefficients that have to be set. $w$ is a weighting factor such that $0 \leq w \leq 1$, which balances the contribution of the region- and edge-based terms in Equation (4) that were normalized between 0 and 1. A higher value of $w$ allows one to associate a higher weight to the region-based term, whereas a smaller value of $w$ yields a higher weight associated to the edge-based term. Since the edge-based segmentation is believed to be more effective than the region-based one when the reconstructed contours is close to the contour reconstructed at the end of the previous iteration, it is possible to recursively adapt the weighting factor $w$ in consecutive iterations of the algorithm. In this study, we advocate to apply a decreasing function $w(t)$ over time, in order to let the active contours be driven by the region-based term in first iterations, when larger changes of the reconstructed contour in consecutive iterations are expected, and by the edge-based term for the final refinement of the segmentation when small changes are expected from one iteration to the next one. The segmen-
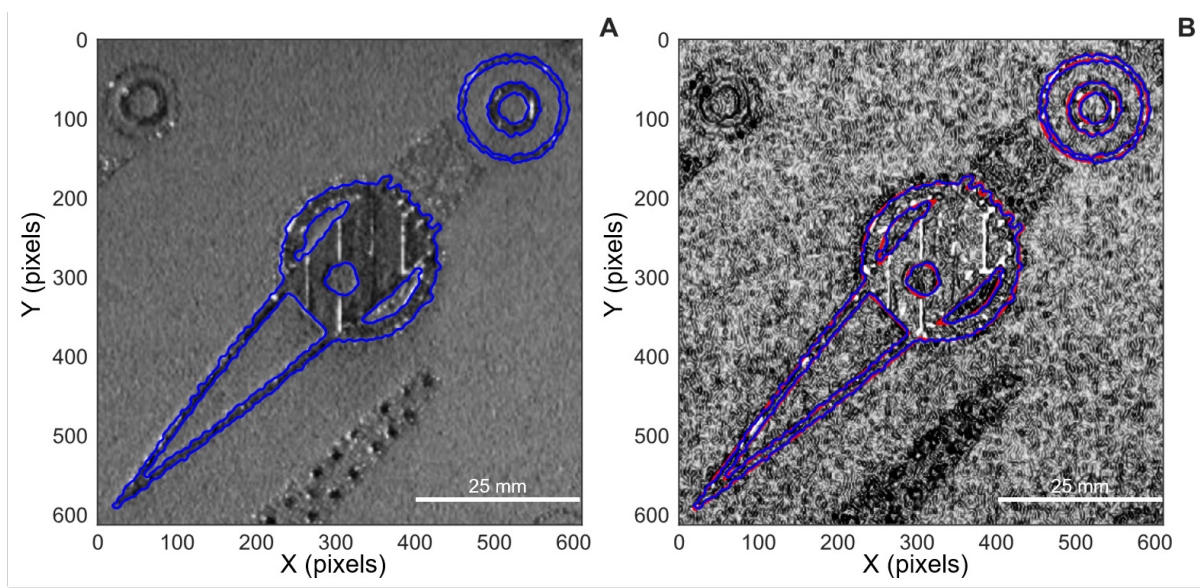

Figure 9: Segmentation of the build part (A) and value of the speed image (B) after removing the bright spots. The blue lines represent the segmented objects, while the red lines represent the nominal mask

tation of one sample image, after the pre-processing steps described in Section 3.1, along with 
the corresponding speed map, is shown in Figure 9. By comparing the contour reconstruction in Figure 9 with the ones presented in Figure 1, it is possible to highlight the actual benefits of the proposed active contours methodology compared with region-based algorithms previously applied in the literature. This approach is particularly effective in reconstructing also critical features like straight and curved thin walls.

\subsection{Deviation map estimation}

The proposed process monitoring approach relies on the estimation of the deviation between the in-situ reconstructed contour of the slice, representative of the as-built geometry in the current layer, and its nominal contour, representative of the as-designed geometry. It is proposed to create a deviation map that associates to each pixel in the image a scalar value that is the distance between that pixel and the closest pixel belonging to the nominal contour. This allows associating a deviation value to each pixel belonging to the in-situ reconstructed contour. An example of distance map with a range of \pm 20 pixels from the null deviation is shown in Figure 10, superimposed either to the nominal contour and the in-situ reconstructed one.

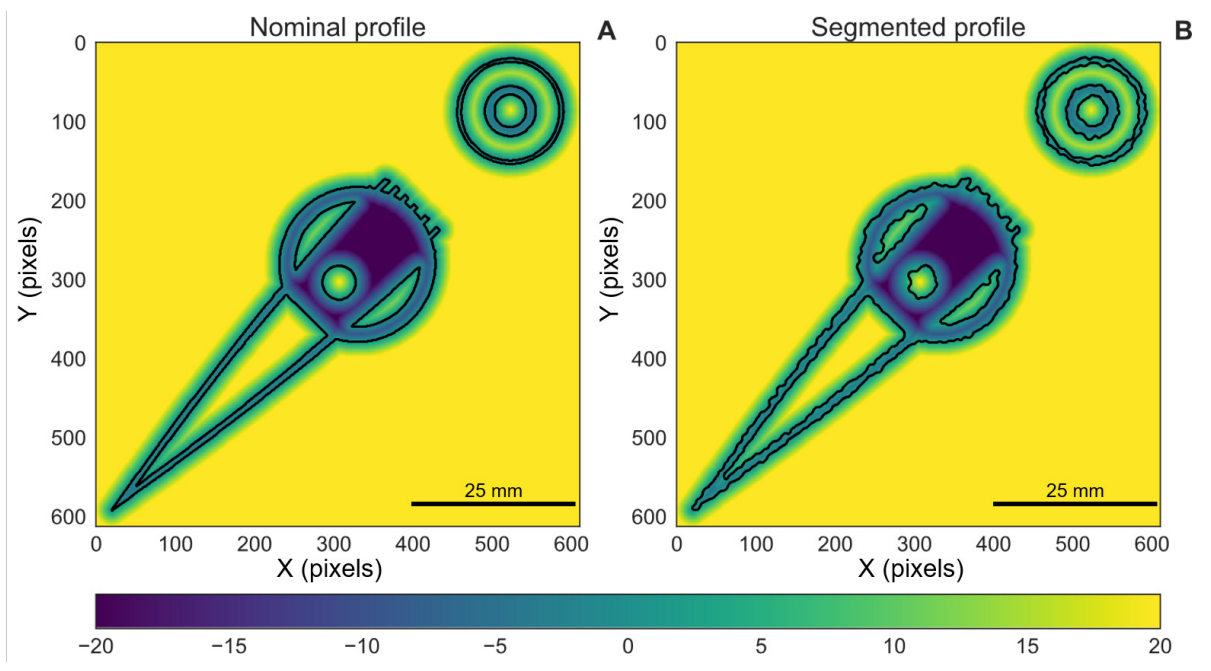

Figure 10: Nominal contour (A) and in-situ reconstructed contour (B) superimposed to the estimated deviation map. The color bar corresponds to the signed distance from the closed pixel along the nominal contour

Since the goal of the proposed approach is to identify abrupt deviations from the nominal contour, it is possible to synthesize the deviation pattern for each in-situ reconstructed contour into a global deviation metric for the design of a statistical process monitoring tool. To this aim, a synthetic index based on the analysis of extreme values is proposed. Let $d_{i}(j)$ be the Euclidean distance between the $j$-th pixel of the reconstructed contour and the closest point belonging to the nominal contour for the i-th layer. For sake of simplicity, in this notation additional sub-scripts indicating different parts printed in the same layer is skipped. The minimum and maximum deviations from the nominal contour in the i-th layer can be estimated as follows:

$$
d_{i}^{\max }=\max _{j} d_{i}(j), \quad d_{i}^{\min }(j)=\min _{j} d_{i}(j) .
$$


The proposed synthetic index is the maximum absolute value of the two extreme deviations along the reconstructed contour:

$$
d_{i}^{\operatorname{minmax}}=\max \left\{\left|d_{i}^{\max }\right|,\left|d_{i}^{\min }\right|\right\} .
$$

The use of the absolute value in the definition of $d_{i}^{\operatorname{minmax}}$ allows one to signal any extreme deviation from the nominal geometry regardless of the direction of the deviation itself. If $d_{i}^{\operatorname{minmax}}=$ $\left|d_{i}^{\text {min }}\right|$, the most extreme deviation is towards the inside of the foreground region, which corresponds to a portion of the reconstructed slice that is missing. If $d_{i}^{\operatorname{minmax}}=\left|d_{i}^{\max }\right|$, the most extreme deviation is towards the outside of the foreground region, which corresponds to a portion of the reconstructed slice that is larger than the nominal shape. Both these two conditions are of interest from a process monitoring perspective, as a lack of material or an excess of material in the slice are both potential symptoms of an anomaly. Section 5 presents a comparison against other conventional statistical indices (mean deviation and standard deviation) to highlight the benefits of using extreme value statistics.

\subsection{Statistical process monitoring}

Since the goal of the proposed method is to automatically signal only anomalous large deviations from the nominal shape, a one-sided control chart is proposed. It allows comparing the $d_{i}^{\operatorname{minmax}}$ index value in the $i-t h$ layer with an upper control limit (UCL) and, whenever the computed value violates the limit, an alarm is signalled. The control limit can be estimated considering that the monitored index is the extreme value within a set of random variables. One way to model the distribution of an extreme statistic involves referring to the Gumbel distribution [29], whose probability density distribution is:

$$
f(x)=\exp \left\{\frac{x-\mu}{\sigma}+\exp \left\{-\frac{x-\mu}{\sigma}\right\}\right\}
$$

where $\mu$ is the location parameter and $\sigma$ is the scale parameter. These two parameters can be estimated using the maximum likelihood (ML) estimation [30]. Let $n$ be a small number of initial observations for the $d^{\operatorname{minmax}}$ index, i.e., a small number of initial layers, the ML estimations of the location $(\mu)$ and the scale $(\sigma)$ parameters can be found maximizing:

$$
\begin{aligned}
& L\left(X_{1}, X_{2}, \ldots, X_{n} \mid \mu, \sigma\right)= \\
& \prod_{i=1}^{n} \exp \left\{\frac{x_{i}-\mu}{\sigma}+\exp \left\{-\frac{x_{i}-\mu}{\sigma}\right\}\right\}
\end{aligned}
$$

with respect to $\mu$ and $\sigma$. By setting a false alarm rate (also known as Type I error) $\alpha$, the UCL of the $d^{\text {minmax }}$ control chart is defined as the $1-\alpha$ percentile of the Gumbel distribution in the first $n$ layers:

$$
U C L=f_{1-\alpha}(x)
$$

being $x=d^{\text {minmax }}$. However, the parameters and the control limit estimated in the first $n$ layers can not be fully representative of the evolution of the monitored statistic in the following layers. Indeed, the deviation map may be affected by the geometry itself, as critical features like acute corners and thin walls may be more difficult to reconstruct than simpler features, and the geometry of the slice may considerably change along the build direction. This implies that the natural 
variability of the deviation synthesized into the $d^{\operatorname{minmax}}$ index may be influenced by the layer-bylayer evolution of the slice geometry. In this scenario, traditional statistical process monitoring approaches, see for example Montgomery [31], based on a fixed training phase may be not fully effective, as they rely on the assumption of temporal independence of the monitored statistic. To cope with the geometry-dependent and adaptive nature of the monitored index, an adaptive monitoring scheme based on a so-called moving window updating procedure is proposed. Let $U C L_{0}, \mu_{0}$ and $\sigma_{0}$ be the parameters estimated on the basis of the first $n$ layers. The control limit $U C L_{0}$ is used to determine if the deviation from the nominal geometry in the $(n+1)-t$ th layer is in-control or not. If $d_{n+1}^{\operatorname{minmax}}>U C L_{0}$ an alarm is signalled. If $d_{n+1}^{\operatorname{minmax}} \leq U C L_{0}$, the parameters of the control chart are recursively updated by maximizing the likelihood of the most recent $n$ layers, leading to new estimates of $U C L_{1}, \mu_{1}$ and $\sigma_{1}$. Such updating scheme is known as "moving window", where a fixed number $n$ of most recent observations is used to determine if the following observation is in-control or not, and older observations are discarded from the updating scheme, as they are deemed to be less representative of the current process pattern. This updating operation is repeated for each new layer unless an alarm is signalled. Thanks to this approach, a moving window of size $n$ is used to determine whether the current value of $d_{i}^{\operatorname{minmax}}$ is anomalous with respect to the natural variability of the same descriptor in the $n$ most recent layers.

\section{Discussion of results}

\subsection{Preliminary test under in-control conditions}

The proposed approach was first tested by means of a build including 3 simple cylindrical specimens of $12 \mathrm{~mm}$ diameter and $20 \mathrm{~mm}$ height produced in Ti6A14V with the EOS M290 system. Default and fixed process parameters for the Ti6Al4V powder were used. A total of 333 layers were monitored for each specimen. The powder bed camera embedded into the EOS M290 system with default illumination and image acquisition settings defined by the system developer was used (see Section 2). The aim of this first test was to characterize the performance of the methodology in terms of false alarm rates, as the cylindrical specimens were representative of regular and in-control shapes produced without distortions. A Python implementation of the morphological active contours algorithm proposed by Márquez-Neila et al. [32] was then implemented. Examples of the segmentation of the analyzed specimens are shown in Figure 11. Three differ-
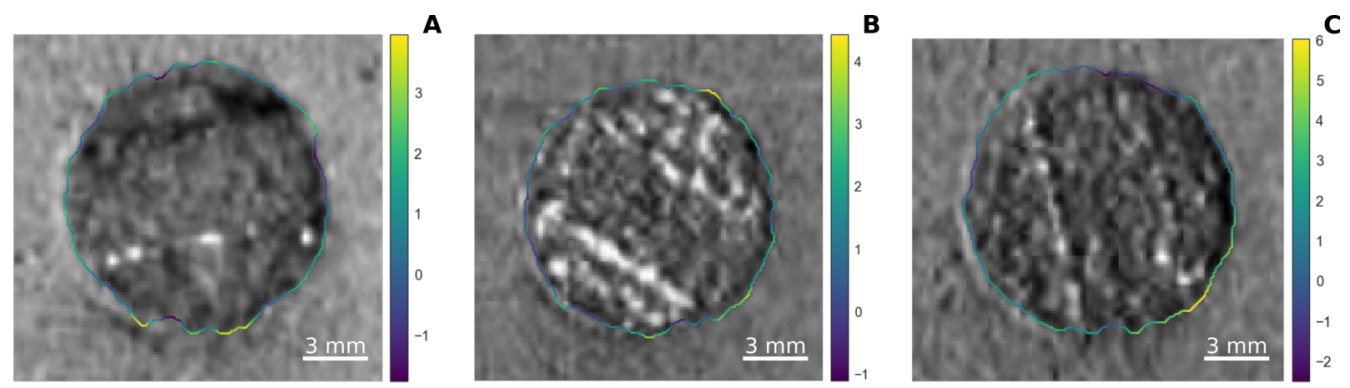

Figure 11: Example of segmentation of three different circular slices of cylindrical specimens in one layer; the colour map of the reconstructed contour indicates the local deviation - in pixels - from the nominal contour

ent values of the moving window size for the design of the proposed control chart were tested, 
namely $n=20, n=30$ and $n=40$. The target false alarm rate was set at $\alpha=1 \%$. Table 1 shows the resulting false alarm rates for all the specimens and for different sizes of the moving window. Table 1 also compares the performances achieved by using the proposed statistic $d^{\text {minmax }}$ against two other possible descriptors, namely the sample mean $\mu_{d}$ and the sample standard deviation $\sigma_{d}$ of the deviation values along the reconstructed contour. The control charts for the latter two indexes were designed by setting the control limits in correspondence of quantiles of their respective distributions and applying the same moving window updating scheme proposed for the $d^{\operatorname{minmax}}$ index.

\begin{tabular}{l|c|c|c|c|c|c|c|c|c}
\hline \multirow{2}{*}{$\begin{array}{c}\text { Window } \\
\text { size }\end{array}$} & \multicolumn{3}{c|}{$\begin{array}{c}\text { Sample } \\
\text { number }\end{array}$} & \multicolumn{3}{c|}{$\begin{array}{c}\text { Sample } \\
\text { number }\end{array}$} & \multicolumn{3}{|c}{$\begin{array}{c}\text { Sample } \\
\text { number }\end{array}$} \\
\cline { 2 - 11 } & 1 & 2 & 3 & 1 & 2 & 3 & 1 & 2 & 3 \\
\hline 20 & $0.3 \%$ & $1.2 \%$ & $0.6 \%$ & $1.5 \%$ & $2.1 \%$ & $1.5 \%$ & $2.4 \%$ & $3.3 \%$ & $0.6 \%$ \\
30 & $0.3 \%$ & $1.2 \%$ & $0.6 \%$ & $0.6 \%$ & $1.5 \%$ & $0.9 \%$ & $3.0 \%$ & $3.3 \%$ & $1.5 \%$ \\
40 & $0.3 \%$ & $1.2 \%$ & $0.3 \%$ & $0.6 \%$ & $1.5 \%$ & $0.9 \%$ & $6.6 \%$ & $3.6 \%$ & $1.5 \%$ \\
\hline
\end{tabular}

Table 1: False alarm rates as a function of the moving window size for different monitored indexes

Table 1 shows that the control chart based on the standard deviation yields a higher rate of false alarms than the ones based on $d^{\operatorname{minmax}}$ and $\mu_{d}$. This may be caused by the violation of the independent and identically distributed data assumption. All the statistics used to quantify the deviation from the nominal shape in each layer are auto-correlated, with a non-stationary auto-correlation pattern that follows the layerwise variation of the slice geometry. The standard deviation is particularly sensitive to these layer-by-layer variations. Although the sample statistics were normalized with respect to the contour length, a change in the geometry affects the distribution of the deviations from the nominal, especially its variability. The sample mean and the proposed index are more robust to layerwise geometry variations. The window size in the considered range has no significant effect on the false alarm rate when the $d^{\text {minmax }}$ index is monitored, whereas a slightly larger false alarm rate was observed when the $\mu_{d}$ index was monitored with $n=20$. This is caused by the fact that a larger window size $n$ is needed to achieve the target false alarm rate $\alpha$ when the standard deviation is used as synthetic index to monitor the deviation between the reconstructed and the nominal contours. These results highlight that the proposed monitoring scheme is suitable to keep the false alarm rate close to the target value for simple and in-control geometries.

\subsection{Monitoring of complex shapes}

The proposed approach was then applied to the case study involving the complex shapes introduced in Section 2, with a further comparison between adaptive control charts based on $d^{\operatorname{minmax}}$ and $\mu_{d}$. In this case, the moving window size was set at $n=30$. Figure 12 shows the moving window control chart applied to the sample 1A. The control limits are shown as dashed red lines, whereas the vertical green dashed line separates the first $n=30$ layers, where a constant control limit was applied, from the following layers where adaptive control limits were used. The control chart in Figure 12 shows only one out-of-control layer: the corresponding image segmentation is shown in Figure 13, where the colors of pixels belonging to the reconstructed contours 


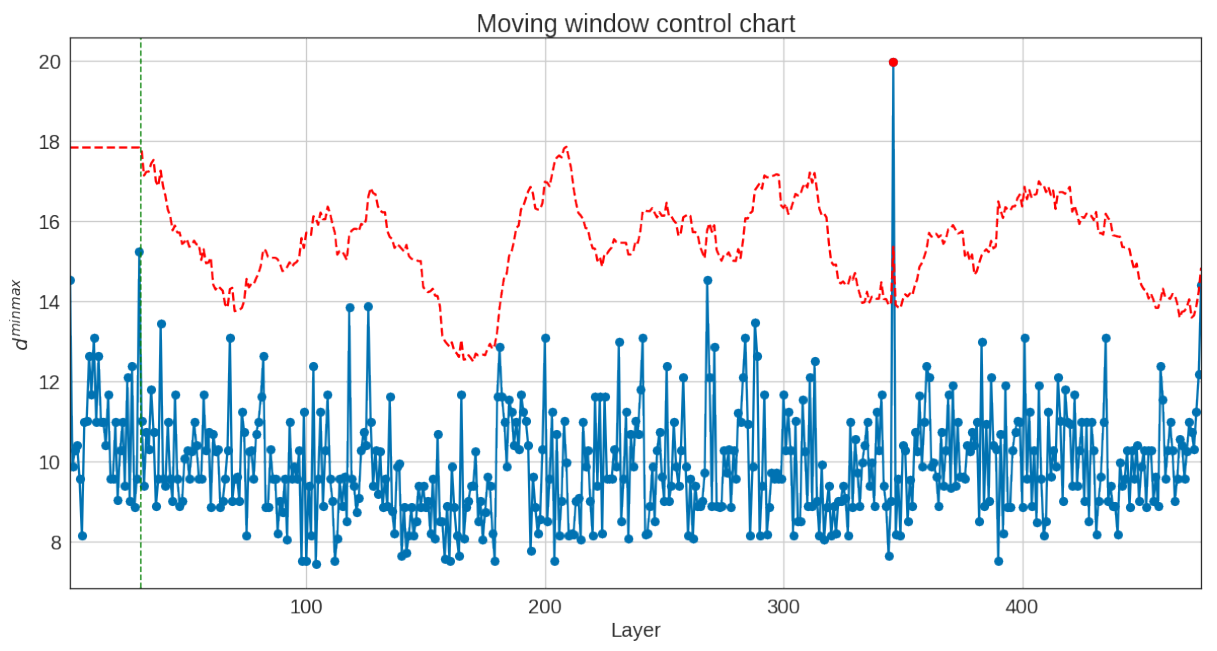

Figure 12: Proposed control chart applied to sample 1A

represent the corresponding deviation values. In the layer depicted in Figure 13, an actual distortion occurred. Due to the thermal stresses in a circular overhang region of the specimen, a partial detachment of the part from the supports occurred during the build. This caused a curling of the border of the overhang region, leading to an out-of-plane distortion that was properly captured by the proposed approach. The same distortion occurred in sample $1 \mathrm{~B}$, which has the

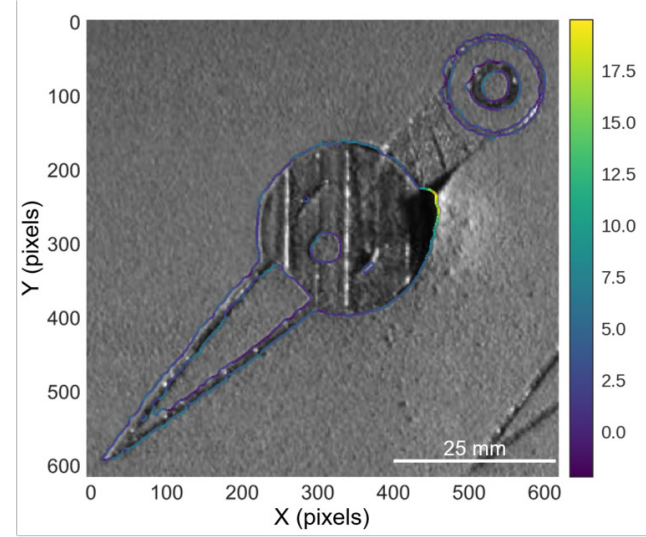

Figure 13: Example of image segmentation for the layer signalled as out-of-control by the control chart for sample 1A; the color bar indicates the deviations from the nominal geometry along the reconstructed contour

same orientation of sample 1A. Figure 14 shows the proposed control chart applied to sample 1B. In this second example, after a first macro-deviation signalled in layer 348 and shown in Figure 15A, analogous to the one occurred in sample 1A, another deviation related to the same thermal-stress induced distortion was signalled few layers later. This second deviation is depicted in Figure 15B, where a curling of the overhang region created a super-elevated area connected to the contour of the scanned slice. This area was partially included into the contour reconstruction 


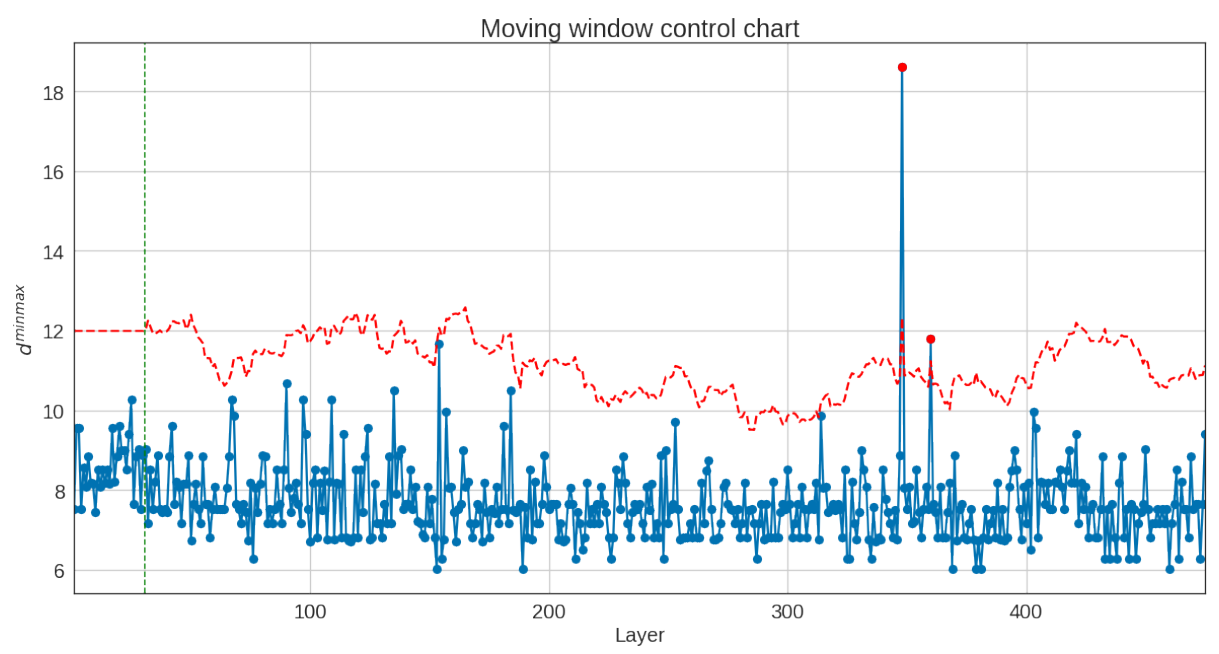

Figure 14: Proposed control chart applied to sample 1B
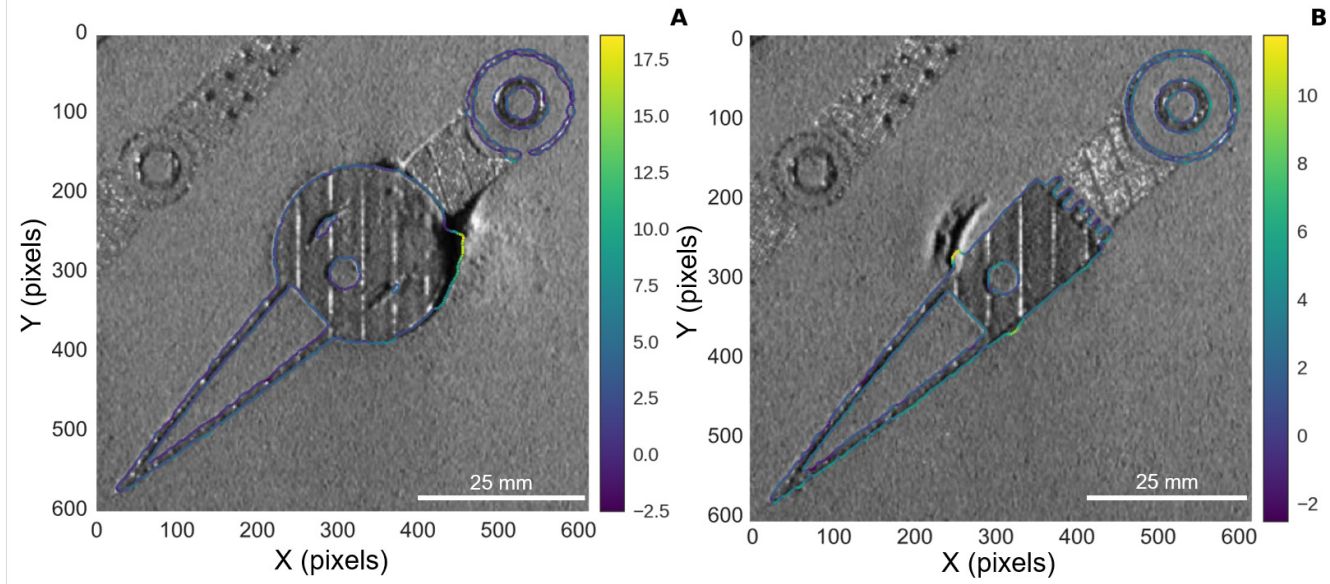

Figure 15: Examples of image segmentations for the layers signalled as out-of-control by the control chart for sample $1 \mathrm{~B}$; the color bar indicates the deviations from the nominal geometry along the reconstructed contour

and then signalled by the control chart. This second anomaly, properly signalled by the proposed approach, did not occur in sample 1A. Figure 16 shows a detail of an ex-situ reconstruction of the deviation between the outer geometry of sample 1A measured by means of X-ray computed tomography (CT) and its nominal geometry. The voxel size of the reconstructed CT volume was $39.48 \mu \mathrm{m}$. Figure 16B shows a larger distortion between the measured geometry and the nominal one in correspondence of the circular overhang portions of the sample. During the build, these distortions caused a partial detachment from supports in both sample $1 \mathrm{~A}$ and $1 \mathrm{~B}$, leading to the deviations reported by the proposed approach based on layerwise images. With the available spatial resolution, the signalled alarms corresponded to deviations in the order of about 1.5 $3 \mathrm{~mm}$. These deviation entities were in agreement with the distortions observed via X-ray CT. 

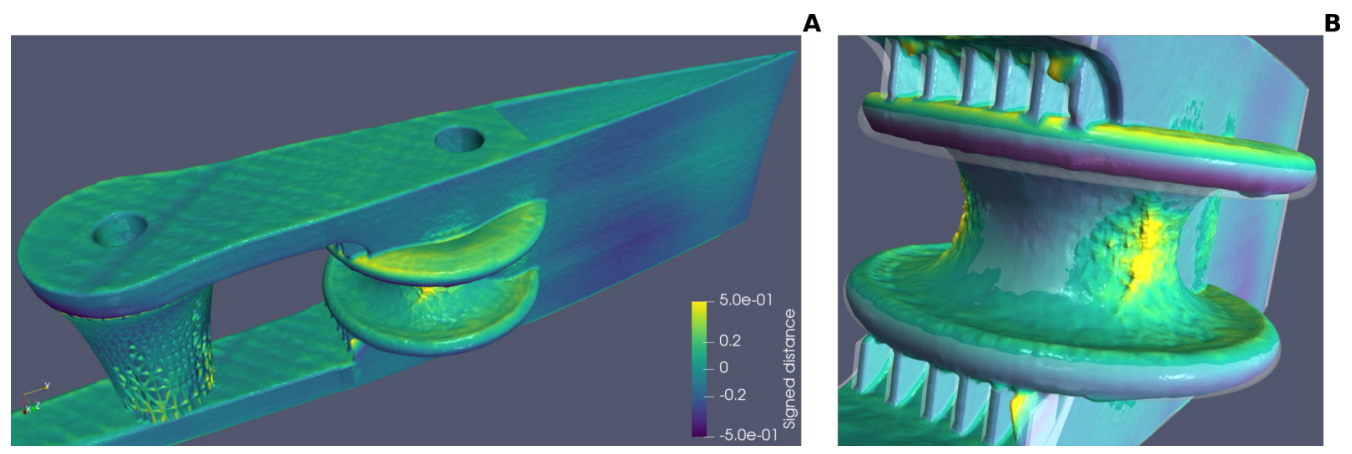

Figure 16: Signed distance between the measured geometry and the nominal geometry of sample 1A (A) with a magnification of the error in the region where the larger distortion occurred (B). The signed distance is expressed in $\mathrm{mm}$

Figure 17 shows the control chart on the average deviation, $\mu_{d}$, applied to sample $1 \mathrm{~A}$. Monitor-

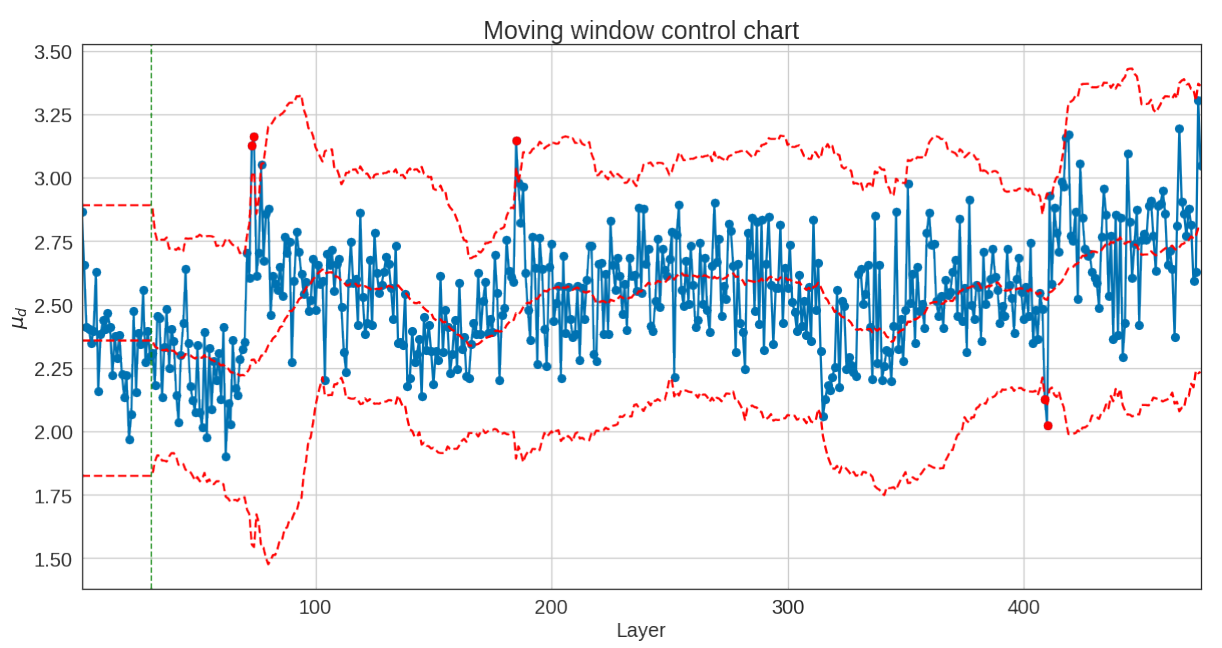

Figure 17: Control chart for sample 1A based on the mean deviation $\mu_{d}$

ing the average deviation instead of the maximum deviation does not allow the detection of the occurred distortion. Indeed, the mean deviation index, $\mu_{d}$, is much less sensitive to the occurred distortion than the absolute maximum deviation, $d^{\operatorname{minmax}}$. This confirms the benefit of monitoring the extreme deviation rather than its average value. Moreover, Figure 17 shows that the control chart based on $\mu_{d}$ applied to sample $1 \mathrm{~A}$ signalled other four alarms that were not related to actual distortions, and hence they can be considered as false alarms. This further confirms the higher robustness of the proposed approach based on the $d^{\operatorname{minmax}}$ index.

Table 2 summarizes the performances of the proposed approach in terms of false alarm rates and actual distortion detection capabilities. The false alarm percentage was computed as the number of false alarms divided by the number of in-control layers, while the actual distortion detection was computed as the percentage of the actual distortions reported as out-of-control. Actual distortions occurred only in samples $1 \mathrm{~A}$ and $1 \mathrm{~B}$, because of their orientation, and they 


\begin{tabular}{l|rrrrrr}
\hline & \multicolumn{6}{|c}{ Sample } \\
\cline { 2 - 7 } & \multicolumn{1}{|c}{$1 \mathrm{~A}$} & $1 \mathrm{~B}$ & $2 \mathrm{~A}$ & $2 \mathrm{~B}$ & $1 \mathrm{C}$ & $2 \mathrm{C}$ \\
\hline False alarm & $0 \%$ & $0 \%$ & $0.2 \%$ & $0 \%$ & $0 \%$ & $0.2 \%$ \\
Actual distortion detection & $100 \%$ & $100 \%$ & - & - & - & - \\
\hline
\end{tabular}

Table 2: Summary of the performances of the proposed approach in terms of false alarms and detection of actual distortions

were properly detected. No distortion occurred in sample $1 \mathrm{C}$, although it was produced with the same orientation of the former two. No distortion occurred also in samples $2 \mathrm{~A}$ and $2 \mathrm{~B}$. A false alarm rate in sample $2 \mathrm{~A}$ and sample $2 \mathrm{C}$ correspond to a violation of the control limit in one layer out of 475 for each sample. These two violations are depicted in Figure 18. They were labelled as false alarms because they caused no visible defect on the final parts. However, Figure 18 shows
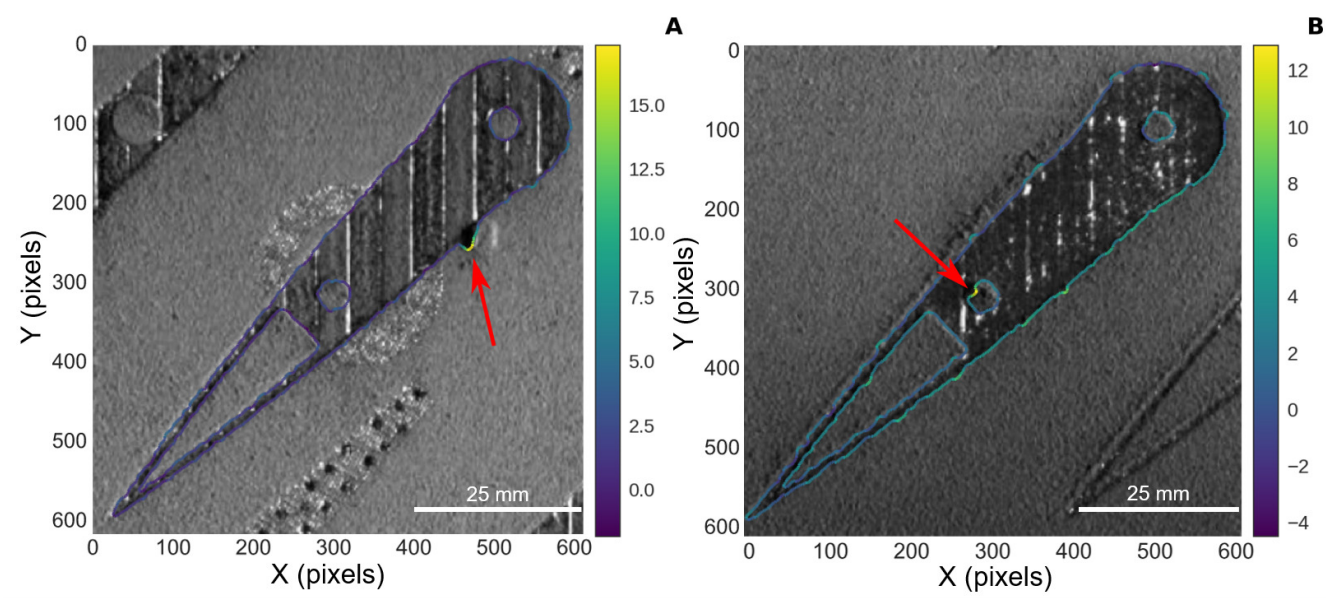

Figure 18: False alarms signalled in samples 2A (A) and 1C (B)

that some dark regions connected to the contour of the slice were actually present in the layerwise images, and they caused an alarm. We believe that those darker areas are shadows projected by out-of-plane irregularities in the layer. Indeed, the low angle illumination integrated into the EOS system used in this study emphasizes surface irregularities, but it is known from previous studies (e.g., Caltanissetta et al. [12]) that it is a non-optimal lighting condition for image segmentation. Although detecting the presence of large shadowed areas along the contour of the slice may be of interest to identify severe surface irregularities, better segmentation performances may be achieved by using a dark-field illumination. Generally speaking, there is a cost-benefit trade-off between using illumination and machine vision equipment already available in the machine (like in this study) or enhancing the performances at the cost of modifying and tuning the equipment. These examples show that the proposed control chart can be used to automatically detect potential anomalies on a layerwise basis. The signalled alarm may be analysed in real-time through the human-machine interface to support the operator in deciding whether a process interruption is needed, or just at the end of the build, as a diagnostic tool to support following qualification 
steps. The method could be combined with other existing powder bed monitoring tools aimed at detecting contaminations in the powder bed and errors in the powder bed recoating.

\section{Conclusions and future developments}

In-situ sensing and monitoring methodologies have been gaining a continuously increasing attention in L-PBF to anticipate the detection of defects during the process, and to support the qualification of additively produced parts. The layerwise production paradigm enables the opportunity to gather a large amount of data while the part is being produced.

This study proposed a methodology to take advantage of layerwise imaging to identify, in an automated way, geometrical distortions in terms of macro-deviations from the nominal geometry in each layer. The proposed approach combines a robust image segmentation technique based on active contours with an in-process adaptive control chart to automatically signal abrupt deviations from the nominal shape taking into account the natural geometry evolution from one layer to another. The method was applied to in-situ monitoring of both simple cylindrical specimens and complex test specimens including various critical geometrical features. The algorithm was implemented by using an industrial equipment, without any modification of the existing hardware. The results showed that the proposed approach is suitable to properly detect the contours of the printed slice and to identify actual modifications of the in-situ reconstructed contours, either caused by local contaminations on the layer or by actual distortions in the part. Such capability is particularly attracting from an industrial implementation viewpoint, as almost all industrial L-PBF systems are equipped with powder bed cameras that can be used for the same purpose. The layerwise geometry segmentation enables also the reconstruction of a 3D surface of the whole part at the end of the process. The method is suitable to detect geometrical distortions that affect the currently monitored layer. Therefore it is not suitable to detect deviations originating after the production of the monitored layer and/or distortions whose severity is too low compared to the camera resolution and the measurement uncertainty. Although the limitations imposed by the layerwise monitoring paradigm can not be overcome, the minimum size of detectable distortions can be improved by using an imaging setup involving a higher resolution and ad-hoc illumination conditions. Future analysis may be devoted to characterize the performances of the in-situ geometry measurement and to this aim reference artifacts like the one developed by NIST [33] can be used, including several different geometrical features. However, it is worth noticing that part dimensions and geometries measured in-situ are not fully representative of the final dimensions and geometry, because of shrinkages and thermal stress-induced distortion that may occur after the monitored layer has been produced. However, if a major departure from the expected shape is observed in one layer, it is worth signalling to let the operator decide if actions are needed or, at least, to support following post-process qualification steps.

Additional research efforts may be devoted to the in-line geometry reconstruction methodology and, in particular, to the estimation of the local uncertainty of the reconstruction, which is currently not available with segmentation methods based on level set theory. Further experiments may be needed to test the robustness of the proposed approach in the presence of different geometries and different types of defects. It is also interesting to investigate different illumination and image acquisition settings as they may have an effect on the in-situ geometry reconstruction performances. Indeed, a relevant aspect for the industrial use of in-line monitoring tools involves the general validity of the proposed methodology and its machine-to-machine transferability, which can be investigated in future studies. 


\section{Acknowledgements}

The authors are grateful to BeamIT SpA and EOS GmbH, which provided the facilities and system to carry out the experimentation. The authors are also grateful to Eng. Stefano Grulli, who designed the samples used in the real case study. LP and PJS acknowledge the UKs Engineering and Physical Sciences Research Council (EPSRC) funding the grant Ref. EP/R024162/1. MG and BMC acknowledge the Italian Ministry of Education, University and Research for the support provided through the Project "Department of Excellence LIS4.0 - Lightweight and Smart Structures for Industry 4.0". MG and BMC's research was also supported by ACCORDO Quadro ASI-POLIMI "Attività di Ricerca e Innovazione" n. 2018-5-HH.0, collaboration agreement between the Italian Space Agency and Politecnico di Milano.

\section{References}

[1] B. M. Colosimo, Q. Huang, T. Dasgupta, F. Tsung, Opportunities and challenges of quality engineering for additive manufacturing, Journal of Quality Technology 50 (2018) 233-252.

[2] B. M. Colosimo, Modeling and monitoring methods for spatial and image data, Quality Engineering 30 (2018) 94-111.

[3] M. Grasso, B. M. Colosimo, Process defects and in situ monitoring methods in metal powder bed fusion: a review, Measurement Science and Technology 28 (2017) 044005.

[4] B. Foster, E. Reutzel, A. Nassar, B. Hall, S. Brown, C. Dickman, Optical, layerwise monitoring of powder bed fusion, in: Solid Freeform Fabrication Symposium, Austin, TX, Aug, 2015, pp. 10-12.

[5] L. T. Phuc, M. Seita, A high-resolution and large field-of-view scanner for in-line characterization of powder bed defects during additive manufacturing, Materials \& Design 164 (2019) 107562.

[6] T. Craeghs, S. Clijsters, E. Yasa, J.-P. Kruth, Online quality control of selective laser melting, in: Proceedings of the 20th Solid Freeform Fabrication (SFF) symposium, Austin (Texas), 8-10 august, 2011, pp. 212-226.

[7] F. Imani, A. Gaikwad, M. Montazeri, P. Rao, H. Yang, E. Reutzel, Process mapping and in-process monitoring of porosity in laser powder bed fusion using layerwise optical imaging, Journal of Manufacturing Science and Engineering, Transactions of the ASME 140 (2018).

[8] Z. Li, X. Liu, S. Wen, P. He, K. Zhong, Q. Wei, Y. Shi, S. Liu, In situ 3d monitoring of geometric signatures in the powder-bed-fusion additive manufacturing process via vision sensing methods, Sensors 18 (2018) 1180.

[9] L. Scime, J. Beuth, Anomaly detection and classification in a laser powder bed additive manufacturing process using a trained computer vision algorithm, Additive Manufacturing 19 (2018) 114-126.

[10] C. Gobert, E. W. Reutzel, J. Petrich, A. R. Nassar, S. Phoha, Application of supervised machine learning for defect detection during metallic powder bed fusion additive manufacturing using high resolution imaging., Additive Manufacturing 21 (2018) 517-528.

[11] M. Aminzadeh, T. Kurfess, Vision-based inspection system for dimensional accuracy in powder-bed additive manufacturing, in: ASME 2016 11th International Manufacturing Science and Engineering Conference, American Society of Mechanical Engineers Digital Collection, 2016.

[12] F. Caltanissetta, M. Grasso, S. Petrò, B. M. Colosimo, Characterization of in-situ measurements based on layerwise imaging in laser powder bed fusion, Additive Manufacturing 24 (2018) 183 - 199.

[13] Q. Lu, N. Nguyen, A. Hum, T. Tran, C. Wong, Optical in-situ monitoring and correlation of density and mechanical properties of stainless steel parts produced by selective laser melting process based on varied energy density, Journal of Materials Processing Technology 271 (2019) 520-531.

[14] S. Kleszczynski, J. Zur Jacobsmühlen, J. Sehrt, G. Witt, Error detection in laser beam melting systems by high resolution imaging, in: Proceedings of the twenty third annual international solid freeform fabrication symposium, volume 2012, 2012.

[15] J. zur Jacobsmühlen, S. Kleszczynski, D. Schneider, G. Witt, High resolution imaging for inspection of laser beam melting systems, in: 2013 IEEE international instrumentation and measurement technology conference (I2MTC), IEEE, 2013, pp. 707-712.

[16] J. zur Jacobsmühlen, S. Kleszczynski, G. Witt, D. Merhof, Elevated region area measurement for quantitative analysis of laser beam melting process stability, in: 26th International Solid Freeform Fabrication Symposium; Austin, TX, 2015, pp. 549-559.

[17] W. S. Land II, B. Zhang, J. Ziegert, A. Davies, In-situ metrology system for laser powder bed fusion additive process, Procedia Manufacturing 1 (2015) 393-403. 
[18] B. Zhang, J. Ziegert, F. Farahi, A. Davies, In situ surface topography of laser powder bed fusion using fringe projection, Additive Manufacturing 12 (2016) 100-107.

[19] F. Imani, A. Gaikwad, M. Montazeri, P. Rao, H. Yang, E. Reutzel, Layerwise in-process quality monitoring in laser powder bed fusion, in: ASME 2018 13th International Manufacturing Science and Engineering Conference, American Society of Mechanical Engineers Digital Collection, 2018.

[20] M. Aminzadeh, A machine vision system for in-situ quality inspection in metal powder-bed additive manufacturing, Ph.D. thesis, Georgia Institute of Technology, 2016.

[21] C. Li, R. Huang, Z. Ding, J. C. Gatenby, D. N. Metaxas, J. C. Gore, A level set method for image segmentation in the presence of intensity inhomogeneities with application to mri, IEEE transactions on image processing 20 (2011) 2007-2016.

[22] M. Abdelrahman, E. W. Reutzel, A. R. Nassar, T. L. Starr, Flaw detection in powder bed fusion using optical imaging, Additive Manufacturing 15 (2017) 1-11.

[23] S. Liu, Y. Peng, A local region-based chan-vese model for image segmentation, Pattern Recognition 45 (2012) 2769-2779.

[24] R. Szeliski, Image alignment and stitching: A tutorial, Found. Trends. Comput. Graph. Vis. 2 (2006) 1-104.

[25] B. B. Avants, N. J. Tustison, M. Stauffer, G. Song, B. Wu, J. C. Gee, The insight toolkit image registration framework, Frontiers in neuroinformatics 8 (2014) 44

[26] B. W. Silverman, Monographs on statistics and applied probability, Density estimation for statistics and data analysis 26 (1986).

[27] S. Osher, R. Fedkiw, Level Set Methods and Dynamic Implicit Surfaces, Springer Verlag, 2003.

[28] S. Soomro, A. Munir, K. N. Choi, Hybrid two-stage active contour method with region and edge information for intensity inhomogeneous image segmentation, PloS one 13 (2018) 1-20.

[29] E. J. Gumbel, Statistical theory of extreme values and some practical applications: a series of lectures, volume 33 US Government Printing Office, 1948.

[30] S. M. Ross, Introduction to probability and statistics for engineers and scientists, Academic Press, 2014

[31] D. C. Montgomery, Statistical quality control, volume 7, Wiley New York, 2009.

[32] P. Márquez-Neila, L. Baumela, L. Alvarez, A morphological approach to curvature-based evolution of curves and surfaces, IEEE Transactions on Pattern Analysis and Machine Intelligence 36 (2014) 2-17.

[33] S. Moylan, J. Slotwinski, A. Cooke, K. Jurrens, M. A. Donmez, Proposal for a standardized test artifact for additive manufacturing machines and processes, in: Proceedings of the 2012 annual international solid freeform fabrication symposium, Austin, TX, 2012, pp. 6-8.

[34] B. E. Hansen, Lecture notes on nonparametrics, Lecture notes (2009).

\section{Appendix A. Sensitivity analysis for the kernel bandwidth selection}

In this Section, a sensitivity analysis with respect to the Gaussian kernel bandwidth is presented. The kernel bandwidth selection is needed in the proposed pre-processing phase to get rid of pixel intensity discontinuities along the offset between adjacent stripes and other saturated bright spots. Kernel bandwidth values between $0.5 \%$ and $10 \%$ were investigated. For each bandwidth value the resulting segmentation of the bright spots was analyzed. It is also worth mentioning that methods for the automatic selection of kernel bandwidths have been proposed in the literature, although they work better in the presence of uni-modal distributions. In addition to a-priori defined bandwidth values, an automated selection approach based on the so-called normal reference was applied as discussed in Hansen [34]. This result led to a bandwidth very close to $1 \%$. The resulting threshold on pixel intensities and the images after the correction of bright areas are shown in Figures A.19, A.20, A.21 and A.22 for the automated selection method, $10 \%, 5 \%$ and $0.5 \%$ bandwidths, respectively. Generally speaking, the higher is the value of the bandwidth, the higher can be the risk to underestimate the actual bright areas. However, the results showed that no significant difference was observed in the corrected images for all tested bandwidth values. This makes the proposed pre-processing step robust with respect to the kernel density fitting. In this study a bandwidth equal to $1 \%$ was used. 

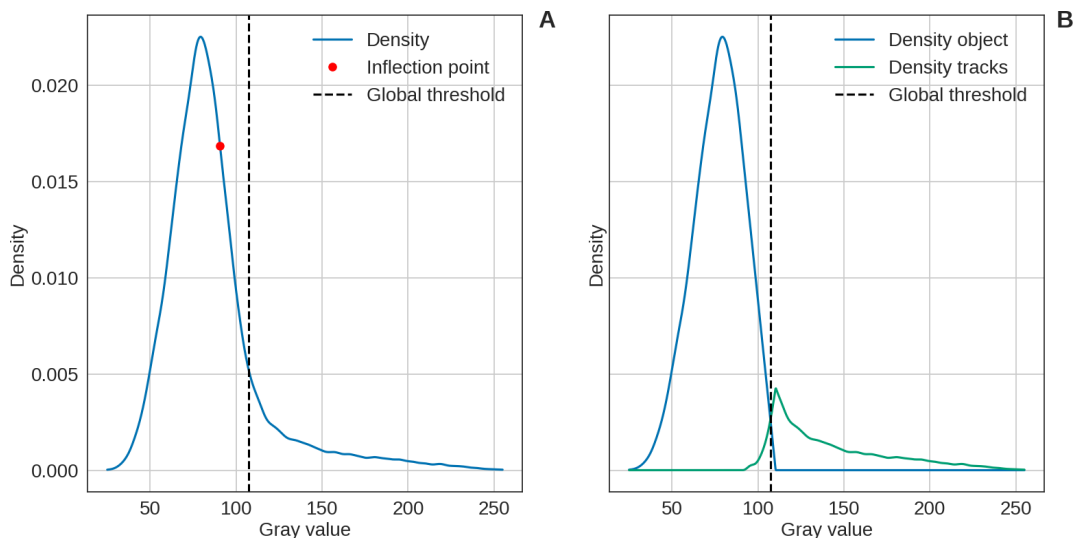

(a) Example of an estimated kernel density function of the pixels within the nominal mask (A), and computed density functions of the dark and bright pixels within the same region, with the indication of the global threshold (B)
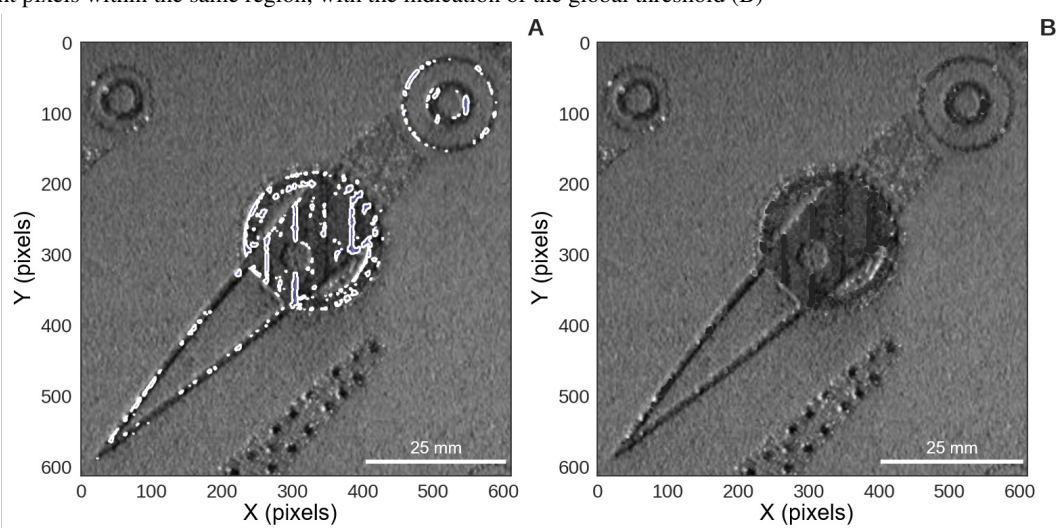

(b) Isolation of bright areas in the foreground region (A) and resulting image after the proposed pixel intensity correction operation (B)

Figure A.19: Post-processing using the automated bandwidth selection method based on normal reference discussed in Hansen [34] 

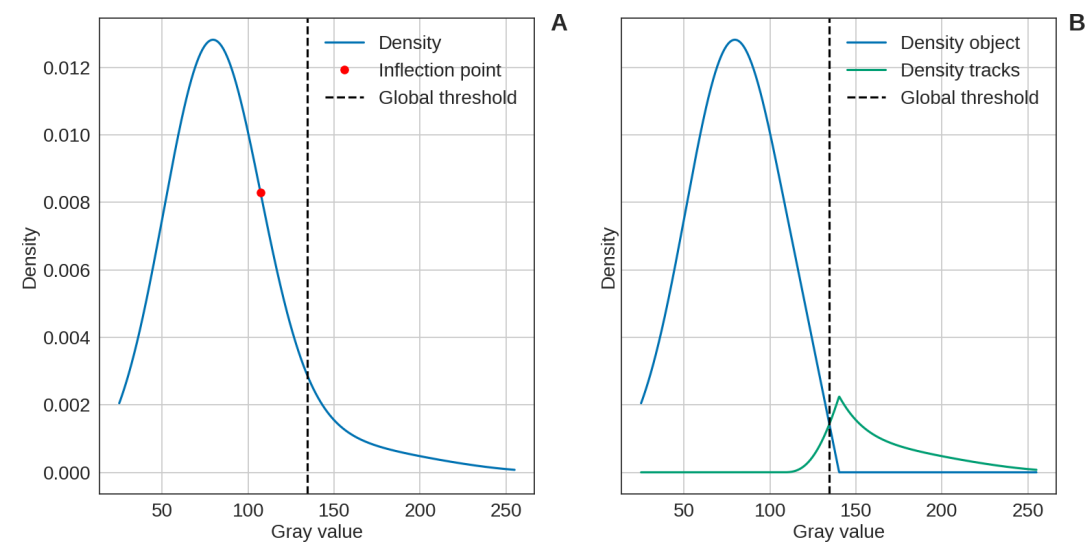

(a) Example of an estimated kernel density function of the pixels within the nominal mask (A), and computed density functions of the dark and bright pixels within the same region, with the indication of the global threshold (B)
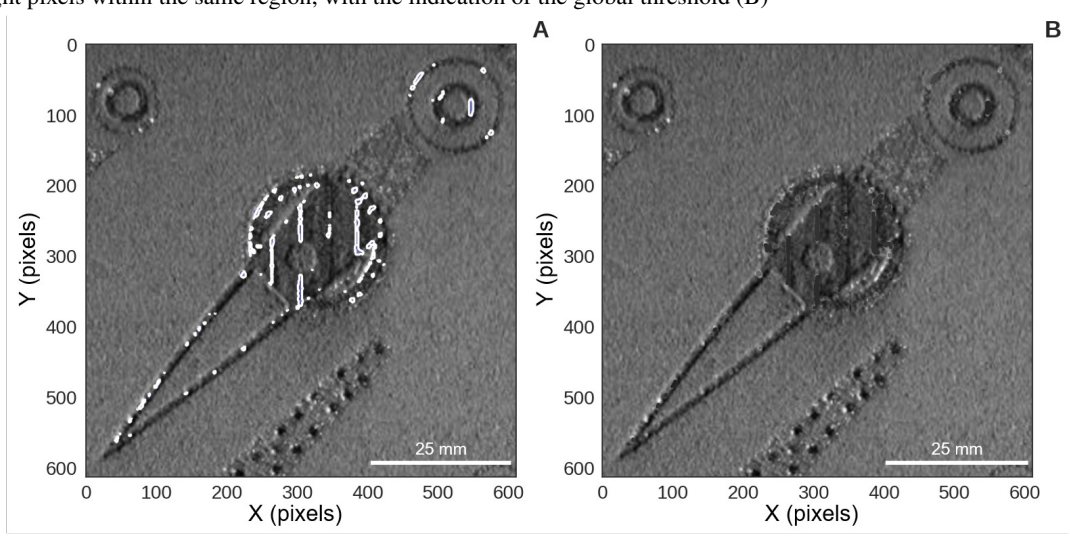

(b) Isolation of bright areas in the foreground region (A) and resulting image after the proposed pixel intensity correction operation (B)

Figure A.20: Post-processing using a threshold equal to the $10 \%$ of the gray values' range 

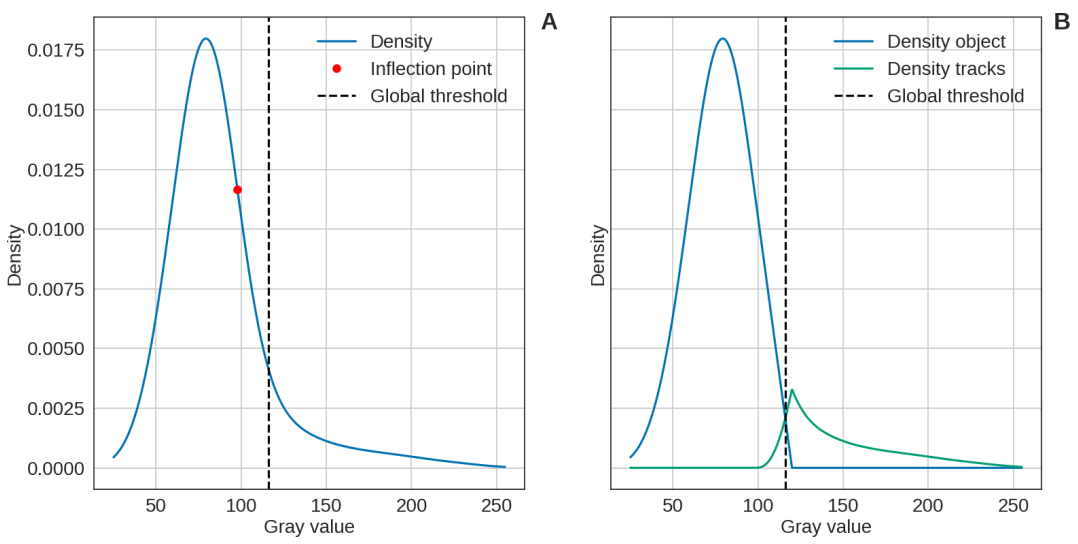

(a) Example of an estimated kernel density function of the pixels within the nominal mask (A), and computed density functions of the dark and bright pixels within the same region, with the indication of the global threshold (B)
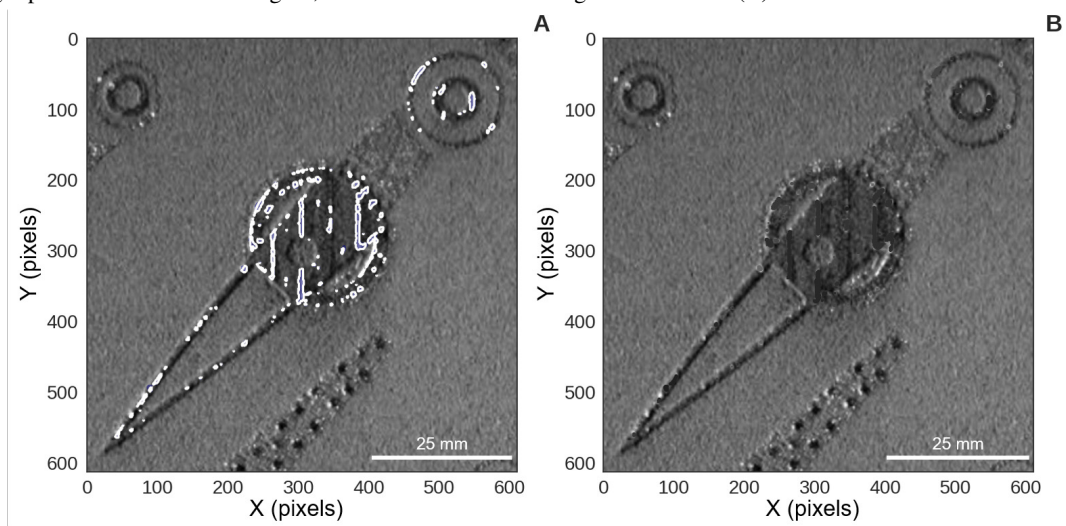

(b) Isolation of bright areas in the foreground region (A) and resulting image after the proposed pixel intensity correction operation (B)

Figure A.21: Post-processing using a threshold equal to the $5 \%$ of the gray values' range 

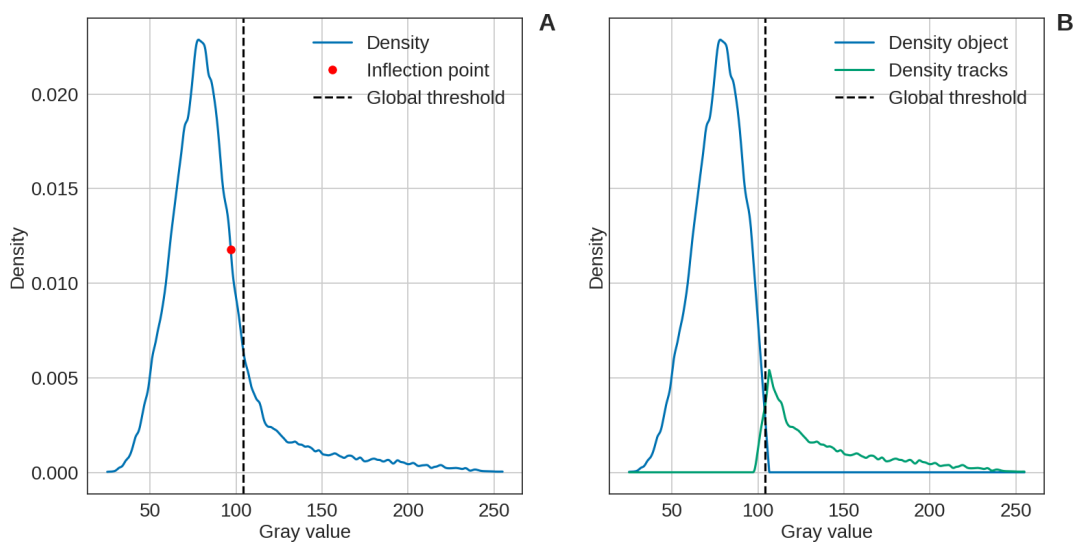

(a) Example of an estimated kernel density function of the pixels within the nominal mask (A), and computed density functions of the dark and bright pixels within the same region, with the indication of the global threshold (B)
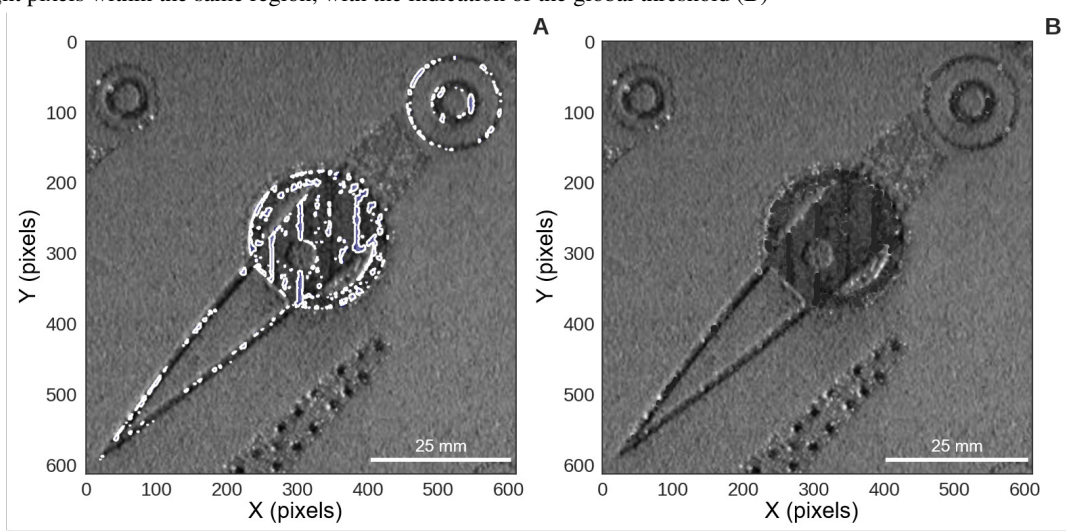

(b) Isolation of bright areas in the foreground region (A) and resulting image after the proposed pixel intensity correction operation (B)

Figure A.22: Post-processing using a threshold equal to the $0.5 \%$ of the gray values' range 